NBER WORKING PAPER SERIES

\title{
THE DEMISE OF WALK ZONES IN BOSTON: PRIORITIES VS. PRECEDENCE IN SCHOOL CHOICE
}

\author{
Umut M. Dur \\ Scott Duke Kominers \\ Parag A. Pathak \\ Tayfun Sönmez \\ Working Paper 18981 \\ http://www.nber.org/papers/w18981
NATIONAL BUREAU OF ECONOMIC RESEARCH
1050 Massachusetts Avenue
Cambridge, MA 02138
April 2013

We thank Kamal Chavda, Carleton Jones, Dr. Carol Johnson, Tim Nicollette, and Jack Yessayan for their expertise and for granting permission to undertake this study. Edward L. Glaeser and Yusuke Narita provided helpful comments. Kominers is grateful for the support of National Science Foundation grant CCF-1216095, an AMS-Simons Travel Grant, and the Human Capital and Economic Opportunity Working Group sponsored by the Institute for New Economic Thinking, as well as for the hospitality of the Harvard Program for Evolutionary Dynamics. Pathak acknowledges support of National Science Foundation grant SES-1056325. The views expressed herein are those of the authors and do not necessarily reflect the views of the National Bureau of Economic Research.

At least one co-author has disclosed a financial relationship of potential relevance for this research. Further information is available online at http://www.nber.org/papers/w18981.ack

NBER working papers are circulated for discussion and comment purposes. They have not been peerreviewed or been subject to the review by the NBER Board of Directors that accompanies official NBER publications.

(C) 2013 by Umut M. Dur, Scott Duke Kominers, Parag A. Pathak, and Tayfun Sönmez. All rights reserved. Short sections of text, not to exceed two paragraphs, may be quoted without explicit permission provided that full credit, including () notice, is given to the source. 
The Demise of Walk Zones in Boston: Priorities vs. Precedence in School Choice

Umut M. Dur, Scott Duke Kominers, Parag A. Pathak, and Tayfun Sönmez

NBER Working Paper No. 18981

April 2013

JEL No. C78,D50,D61,I21

\begin{abstract}
$\underline{\text { ABSTRACT }}$
School choice plans in many cities grant students higher priority for some (but not all) seats at their neighborhood schools. This paper demonstrates how the precedence order, i.e. the order in which different types of seats are filled by applicants, has quantitative effects on distributional objectives comparable to priorities in the deferred acceptance algorithm. While Boston's school choice plan gives priority to neighborhood applicants for half of each school's seats, the intended effect of this policy is lost because of the precedence order. Despite widely held impressions about the importance of neighborhood priority, the outcome of Boston's implementation of a 50-50 school split is nearly identical to a system without neighborhood priority. We formally establish that either increasing the number of neighborhood priority seats or lowering the precedence order positions of neighborhood seats at a school have the same effect: an increase in the number of neighborhood students assigned to the school. We then show that in Boston a reversal of precedence with no change in priorities covers almost three-quarters of the range between $0 \%$ and $100 \%$ neighborhood priority. Therefore, decisions about precedence are inseparable from decisions about priorities. Transparency about these issues - in particular, how precedence unintentionally undermined neighborhood priority—led to the abandonment of neighborhood priority in Boston in[2013.
\end{abstract}

Umut M. Dur

The University of Texas at Austin Department of Economics

1 University Station C3100

Austin, Texas 78712

umutdur@gmail.com

Scott Duke Kominers

Rosenwald Hall 331B

University of Chicago

Chicago, IL 60637

skominers@uchicago.edu
Parag A. Pathak

MIT Department of Economics

50 Memorial Drive

E52-391C

Cambridge, MA 02142

and NBER

ppathak@mit.edu

Tayfun Sönmez

Boston College

Department of Economics

140 Comm. Ave.

Chestnut Hill, MA, 02467

tayfun.sonmez@bc.edu 


\section{Introduction}

School choice programs aspire to weaken the link between the housing market and access to good schools. In purely residence-based public school systems, families can purchase access by moving to neighborhoods with desirable schools. Children from families less able to move do not have the same opportunity. With school choice, children are allowed to attend schools outside their neighborhood without having to re-locate. This may generate a more equitable distribution of school access. Enabling choice requires specifying how school seats will be rationed among students from within and outside of schools' neighborhoods. During the 1970s, court rulings on the appropriate balance of neighborhood and non-neighborhood assignment, often drawn on racial and ethnic lines, had a large impact on the shape of urban America (Baum-Snow and Lutz 2011, Boustan 2012). The debate about the appropriate balance between these two factions continues today within school choice plans.

The initial literature on school choice mechanisms did not explicitly consider the issue of how to specify property rights for school seats. Instead, they were taken as given, and research focused on how property rights are interpreted by the assignment mechanism (Balinski and Sönmez 1999, Abdulkadiroğlu and Sönmez 2003). Efforts in the field have also avoided taking positions on how to endow agents with claims to schools, and instead have advocated for strategy-proof mechanisms, which make it easier for participants to rank schools. ${ }^{1}$ Now, with the growing use of mechanisms based on the student-proposing deferred acceptance algorithm (DA), it is possible to more explicitly consider the role of property rights. ${ }^{2}$ By setting school priorities, such as giving higher claims to sibling or neighborhood applicants, districts using DA can precisely define applicants' property rights in a way that is independent of applicant demand.

Specifying priorities is only one part of determining students' access to schools. Another part involves determining the fraction of seats where the priorities apply. For the last thirteen years, Boston Public Schools (BPS) split schools' priority structures into two equally-sized pieces, with one half of the seats at each school giving students from that school's neighborhood priority, and the other half of the seats not giving neighborhood priority a role. ${ }^{3}$ When students in Boston rank a school in their preference list, they are considered for both types of seats. ${ }^{4}$ The order in which the slots are processed, the slots' precedence order, determines how the seats are filled by applicants.

BPS's current 50-50 seat split emerged out of a city-wide discussion following the end of racial and ethnic criteria for school placement in 1999. Many advocated abandoning choice and returning

\footnotetext{
${ }^{1}$ For example, a December 2003 community engagement process in Boston considered six different proposals for alternative neighborhood zone definitions. However, the only recommendation adopted by the school committee was to switch the assignment algorithm (Abdulkadiroğlu, Pathak, Roth, and Sönmez 2005).

${ }^{2}$ Other mechanisms often lack this complete separation. For instance, in the pre-2005 Boston mechanism, applicants' preference rankings first determined whose claims were justified; priorities were only used to adjudicate claims among equal-ranking applicants.

${ }^{3}$ The 50-50 school seat split was not altered when Boston changed their assignment mechanism in 2005 to one based on the student-proposing deferred acceptance algorithm (Abdulkadiroğlu, Pathak, Roth, and Sönmez 2005, Abdulkadiroğlu, Pathak, Roth, and Sönmez 2006, Pathak and Sönmez 2008).

${ }^{4}$ Throughout this paper, we use slot and seat interchangeably.
} 
to neighborhood schools at that point, but the school committee decided to reduce the fraction of seats where neighborhood, i.e. "walk-zone" priority, applies from $100 \%$ to $50 \%$ of seats within each school. The official policy document states (BPS 1999):

Fifty percent walk zone preference means that half of the seats at a given school are subject to walk zone preference. The remaining seats are open to students outside of the walk zone. RATIONALE: One hundred percent walk zone preference in a controlled choice plan without racial guidelines could result in all available seats being assigned to students within the walk zone. The result would limit choice and access for all students, including those who have no walk zone school or live in walk zones where there are insufficient seats to serve the students residing in the walk zone.

Patterns of parent choice clearly establish that many choose schools outside of their walk zone for many educational and other reasons. [...] One hundred percent walk zone preference would limit choice and access for too many families to the schools they want their children to attend. On the other hand, the policy also should and does recognize the interests of families who want to choose a walk zone school.

The 50-50 slot split was seen as "striking an uneasy compromise between neighborhood school advocates and those who want choice," while the Superintendent hoped that the "plan would satisfy both factions, those who want to send children to schools close by and those who want choice" (Daley 1999).

We begin by investigating the impact of priorities and precedence on assignments in Boston Public Schools. Using data on students' choices and assignments, we examine whether the current BPS mechanism actually represents the intended compromise. We compare the current BPS outcome to two extreme alternatives: one where none of the seats have walk-zone priority, and one where all seats have walk-zone priority. Given the 50-50 split and its motivation, it is natural to expect the outcome under the current BPS mechanism to be close to the midpoint between the two extremal policies. However, Table 1 shows that the outcome of the current BPS mechanism is almost identical to the outcome where walk-zone priority is not used at all.

Despite the perception that walk-zone applicants have been advantaged in the BPS system since 1999, they appear to have little advantage in practice. Only 3\% of Grade K1 (a main elementary school entry point) applicants obtain a different assignment under Boston's current implementation than they would under open competition without walk-zone priority, as indicated in the column labeled $0 \%$ Walk. The difference is as low as $1 \%$ for Grade 6 . Furthermore, this pattern is not simply a feature of student demand. Under the alternative in which all seats have walk-zone priority (labeled 100\% Walk), the number of students assigned to schools in their walk zones increases to $19 \%$ and $17 \%$ for Grades K1 and K2, respectively. Although motivated as a compromise between the two factions, BPS's 50-50 school seat split is significantly closer to open competition than is at first apparent.

Why does Boston's assignment mechanism result in an assignment so close to one without any neighborhood priority, even though half of each school's seats give priority to neighborhood 
students? This paper is about understanding this puzzle. We develop a framework for school choice mechanism design where both priority and precedence play key roles. We show that the division of schools into walk-zone and open priority seats reveals little about the proximity of the outcome to a compromise between the two factions without specifying the precedence order, which determines what happens a student is qualified for both walk-zone and open seats. Building on Kominers and Sönmez (2012), we establish two new comparative statics:

1. Given a fixed slot precedence order, replacing an open slot at a school with a walk-zone slot weakly increases the number of walk-zone students assigned to that school.

2. Given a fixed split of slots into walk-zone and open slots, switching the precedence order position of a walk-zone slot with that of a subsequent open slot weakly increases the number of neighborhood students assigned to that school.

While the first of these results is intuitive, the second one is more subtle. Moreover, they do not follow from earlier monotonicity results in simpler models without slot-specific priority structure (e.g., Balinski and Sönmez (1999)) because they involve simultaneous priority improvements for a large number of students. In a further specialized, two-school model, the types of priority and precedence order changes described above in fact increase neighborhood assignment across all schools. The impact in this case is entirely distributional as both instruments leave the aggregate number of students obtaining their top-choice schools unchanged.

We then empirically examine the extent to which the comparative statics from our simplified model are relevant under the richer priority structure in the Boston's school choice program. After demonstrating that BPS's current implementation of the 50-50 split is far from balancing the concerns of the neighborhood schooling and school choice proponents, we show that an alternative precedence order where open slots are depleted before walk-zone slots results in $8.2 \%$ more students attending walk-zone schools in Grade K1. This represents nearly three-quarters of the maximal achievable difference between completely eliminating walk-zone priority and having walk-zone priority apply at all school seats.

We also examine alternative precedence orders which implement policies between the $0 \%$ Walk and $100 \%$ Walk extremes. Once a preliminary version of this paper was circulated, it entered the policy discussion in Boston where these alternative order proposals were discussed. When our work clarified the role of the precedence order in undermining the intended effects of the 50-50 seat split, BPS completely eliminated neighborhood priority. We describe this and the larger policy discussion in Boston in more detail in Section 4.

This paper contributes to a broader agenda, examined in a number of recent papers, that introduces concerns for diversity into the literature on school choice mechanism design (see, e.g., Budish, Che, Kojima, and Milgrom (2013), Echenique and Yenmez (2012), Erdil and Kumano (2012), Hafalir, Yenmez, and Yildirim (2012), Kojima (2012), and Kominers and Sönmez (2012)). When an applicant ranks a school with many seats, it is similar to expressing indifference among the school's seats. Therefore, our work parallels recent papers examining the implications of indifferences in 
school choice problems (Erdil and Ergin 2008, Abdulkadiroğlu, Pathak, and Roth 2009, Pathak and Sethuraman 2011). However, the question of school-side indifferences, the focus of prior work, is entirely distinct from the issue of indifferences in student preferences. Tools used to resolve indifferences in schools's priorities (e.g., random lotteries) do not immediately apply to the case of student-side indifferences. Another related paper is that of Roth (1985), which shows how to interpret a (Gale and Shapley 1962) college admissions (many-to-one) matching model as a marriage (one-to-one) matching model by splitting colleges into individual seats and assuming that students rank those seats in a given order. Our results show that implementation of this seatsplit approach without attention to precedence can undermine the intentions of priority policies. Finally, this paper builds on the theoretical literature on matching with contracts (Crawford and Knoer 1981, Kelso and Crawford 1982, Hatfield and Milgrom 2005, Ostrovsky 2008, Hatfield and Kojima 2010, Echenique 2012) and the applied motivation shares much with recent work on matching in the military (Sönmez and Switzer 2013, Sönmez 2013).

The paper proceeds as follows. Section 2 introduces the model and illustrates the roles of precedence and priority. Section 3 reports on our empirical investigation of these issues in the context of Boston's school choice plan. Section 4 briefly reviews the current debate in Boston and describes how the present paper played a role in the debate. Section 5 concludes. All proofs are relegated to the Appendix.

\section{Model}

There is a finite set $I$ of students and a finite set $A$ of schools. Each school $a$ has a finite set of slots $S^{a}$. We use the notation $a_{0}$ to denote a "null school" representing the possibility of being unmatched; we assume that this option is always available to all students. Let $S \equiv \bigcup_{a \in A} S^{a}$ denote the set of all slots (excluding those at the null school). We assume that $|S| \geq|I|$, so that there are enough (real) slots for all students. Each student $i$ has a strict preference relation $P^{i}$ over $A \cup\left\{a_{0}\right\}$. Throughout the paper we fix the set of students $I$, the set of schools $A$, the set of schools' slots $S$, and the students' preferences $\left(P^{i}\right)_{i \in I}$.

For any school $a \in A$, each slot $s \in S^{a}$ has a linear priority order $\pi^{s}$ over students in $I$. This linear priority order captures the "property rights" of the students for this slot in the sense that the higher a student is ranked under $\pi^{s}$, the stronger claims he has for the slot $s$ of school $a$. Following the current practice in BPS, we allow slot priorities to be heterogeneous across slots of a given school. A subtle consequence of this within-school heterogeneity is that we must determine how slots are assigned when a student is "qualified" for multiple slots with different priorities at a school. The last primitive of the model regulates this selection: For each school $a \in A$, the slots in $S^{a}$ are ordered according to a (linear) order of precedence $\triangleright^{a}$. Given a school $a \in A$ and two of its slots $s, s^{\prime} \in S^{a}$, the expression $s \triangleright^{a} s^{\prime}$ means that slot $s$ is to be filled before slot $s^{\prime}$ at school $a$ whenever possible.

A matching $\mu: I \rightarrow A$ is a function which assigns a school to each student such that no schools is assigned to more students than its total number of slots. Let $\mu_{i}$ denote the assignment of student 
$i$, and $\mu_{a}$ denote the set of students assigned to school $a$.

Our model generalizes the school choice model of Abdulkadiroğlu and Sönmez (2003) in that it allows for heterogenous priorities across the slots of a given school. Nevertheless, a mechanism based on the celebrated student-proposing deferred acceptance algorithm (Gale and Shapley 1962) easily extends to this model once the choice function of each school is constructed for given slot priorities and order of precedence.

Given a school $a \in A$ with a set of slots $S^{a}$, a list of slot priorities $\left(\pi^{s}\right)_{s \in S^{a}}$, an order of precedence $\triangleright^{a}$ with

$$
s_{a}^{1} \triangleright^{a} s_{a}^{2} \triangleright^{a} \cdots \triangleright^{a} s_{a}^{\left|S^{a}\right|},
$$

and a set of students $J \subseteq I$, the choice of school $\boldsymbol{a}$ from the set of students $\boldsymbol{J}$ is denoted by $C^{a}(J)$, and is obtained as follows: Slots at school $a$ are filled one at a time following the order of

precedence $\triangleright^{a}$. The highest priority student in $J$ under $\pi^{s_{a}^{1}}$, say student $j_{1}$, is chosen for slot $s_{a}^{1}$ of school $a$; the highest priority student in $J \backslash\left\{j_{1}\right\}$ under $\pi^{s_{a}^{2}}$ is chosen for slot $s_{a}^{2}$ of school $a$, and so on.

For a given list of slot priorities $\left(\pi^{s}\right)_{s \in S}$ and an order of precedence $\triangleright^{a}$ at each school $a \in A$, the outcome of the student-proposing deferred acceptance mechanism (DA) can be obtained as follows:

Step 1: Each student $i$ applies to her top choice school. Each school $a$ with a set of Step 1 applicants $J_{1}^{a}$ tentatively holds the applicants in $C^{a}\left(J_{1}^{a}\right)$, and rejects the rest.

Step $\ell$ : Each student who is rejected in Step $\ell-1$ applies to most-preferred school (if any) that has not yet rejected her. Each school $a$ considers its new applicants together with those on hold from Step $\ell-1$, and uses its choice function $C^{a}$ to determine which students are tentatively held and which students are rejected.

The algorithm terminates after the first step in which no students are rejected, assigning students to the schools holding their applications.

\subsection{A Mix of Neighborhood-Based and Open Priority Structures}

In this paper we are particularly interested in the slot priority structure used at Boston Public Schools. There is a master priority order $\pi^{o}$ that is uniform across all schools. This master priority order is obtained via an even lottery and is often referred to as the random tiebreaker. At each school in Boston, slot priorities depend on students' walk-zone and sibling statuses and the random tiebreaker $\pi^{o}$. For our theoretical analysis, we consider a simplified priority structure which only depends on walk-zone status and the random tiebreaker. We show in our empirical analysis of Section 3 that this is a good approximation to the case of Boston Public Schools.

For any school $a \in A$, there is a subset $I_{a} \subset I$ of walk-zone students that is determined with a concrete formula. There are two types of slots: 
1. Walk-zone slots: For each walk-zone slot at a school $a$, any walk-zone student $i \in I_{a}$ has priority over any non-walk-zone student $j \in I \backslash I_{a}$, and the priority order within these two groups is determined with the random tiebreaker $\pi^{o}$.

2. Open slots: $\pi^{s}=\pi^{o}$ for each open slot $s$.

For any school $a \in A$, define $S_{w}^{a}$ to be the set of walk-zone slots and $S_{o}^{a}$ to be the set of open slots. BPS currently uses a priority structure in which half of the slots at each school are walk-zone slots, while the remaining half are open slots. This structure has been historically interpreted as a compromise between the proponents of neighborhood assignment and the proponents of school choice.

An important comparative statics exercise concerns the impact of replacing an open slot with a walk-zone slot under DA for a given order of precedence. One might naturally expect such a change to weakly increase the number of students who are assigned to a walk-zone school. Surprisingly, this is not correct in general, as we show in the next example.

Example 1. There are four schools $A=\{k, l, m, n\}$. Each school has two available slots. There are eight students $I=\left\{i_{1}, i_{2}, i_{3}, i_{4}, i_{5}, i_{6}, i_{7}, i_{8}\right\}$. There are two walk-zone students at each school: $I_{k}=\left\{i_{1}, i_{2}\right\}, I_{l}=\left\{i_{3}, i_{4}\right\}, I_{m}=\left\{i_{5}, i_{6}\right\}$ and $I_{n}=\left\{i_{7}, i_{8}\right\}$. The random tiebreaker $\pi^{o}$ orders the students as:

$$
\pi^{o}: i_{1} \succ i_{8} \succ i_{3} \succ i_{4} \succ i_{5} \succ i_{6} \succ i_{7} \succ i_{2} .
$$

The preference profile is:

\begin{tabular}{cccccccc}
\hline$P^{i_{1}}$ & $P^{i_{2}}$ & $P^{i_{3}}$ & $P^{i_{4}}$ & $P^{i_{5}}$ & $P^{i_{6}}$ & $P^{i_{7}}$ & $P^{i_{8}}$ \\
\hline \hline$k$ & $k$ & $l$ & $l$ & $m$ & $m$ & $n$ & $k$ \\
$l$ & $l$ & $k$ & $k$ & $k$ & $k$ & $k$ & $l$ \\
$m$ & $m$ & $m$ & $m$ & $l$ & $l$ & $l$ & $m$ \\
$n$ & $n$ & $n$ & $n$ & $n$ & $n$ & $m$ & $n$ \\
\hline
\end{tabular}

First consider the case where each school has one walk-zone slot and one open slot. Also assume that the walk-zone slot has higher precedence than the open slot at each school.

The outcome of DA for this case is:

$$
\mu=\left(\begin{array}{cccccccc}
i_{1} & i_{2} & i_{3} & i_{4} & i_{5} & i_{6} & i_{7} & i_{8} \\
k & n & l & l & m & m & n & k
\end{array}\right) .
$$

Observe that six students (i.e. students $\left.i_{1}, i_{3}, i_{4}, i_{5}, i_{6}, i_{7}\right)$ are assigned to their walk-zone schools in this scenario.

Next we replace the open slot at school $k$ with a walk-zone slot, so that both slots at school $k$ are walk-zone slots. Each remaining school has one walk-zone slot and one open slot, with the walk-zone slot having higher precedence than the open slot. 
The outcome of DA for the second case is:

$$
\mu^{\prime}=\left(\begin{array}{cccccccc}
i_{1} & i_{2} & i_{3} & i_{4} & i_{5} & i_{6} & i_{7} & i_{8} \\
k & k & l & m & m & n & n & l
\end{array}\right) .
$$

Observe that five students (i.e. students $i_{1}, i_{2}, i_{3}, i_{5}, i_{7}$ ) are assigned to their walk-zone schools in the second case - the total number of walk-zone assignments decreases when the open slot at school $k$ is replaced with a walk-zone slot.

Nevertheless, as we present next, replacement of an open slot of school $a$ with a walk-zone slot weakly increases the number of walk-zone students assigned to school $a$ (even though it may decrease the total number of walk-zone assignments).

Proposition 1. For any given order of precedence of slots, replacing an open slot of school a with a walk-zone slot weakly increases the number of walk-zone students who are assigned to school a under DA.

When a school district increases the fraction of walk-zone slots, one of the policy motives behind this change is to increase the fraction of students assigned to walk-zone schools. As Proposition 1 shows, replacing an open slot with a walk-zone slot serves this goal through its "first-order effect" in the school directly affected by the change, although the overall effect across all schools might in theory be in the opposite direction. Nevertheless, our empirical analysis in Section 3 suggests that the first-order effect dominates - the overall effect of an increase in the number of walk-zone slots is in the expected direction.

While the role of the number of walk-zone slots as a policy tool is quite clear, the role of the order of precedence is much more subtle. Indeed, the choice of the order of precedence is often considered a minor technical detail - and until the present work, precedence has never entered policy discussions. Nevertheless, precedence impacts outcomes significantly.

Qualitatively, the effect of decreasing the precedence order position of a walk-zone slot is similar to the effect of replacing an open slot with a walk-zone slot. While this may appear counter-intuitive at first, the reason is simple: By decreasing the order of precedence of a walk-zone slot, one increases the odds that a walk-zone student who has a lottery number high enough to make her eligible for both open and walk-zone slots is assigned to an open slot. This in turn increases the competition for the open slots and decreases the competition for walk-zone slots. Our next result formalizes this observation.

Proposition 2. Fix the set of walk-zone slots and the set of open slots at each school. Then, switching the precedence order position of a walk-zone slot of school a with the position of a lowerprecedence open slot weakly increases the number of walk-zone students assigned to school a under $D A$.

Given Example 1, it is not surprising to see that the aggregate effect of lowering walk-zone slot precedence may go against the "first order" effect. We now present a modified version of Example 1 that makes this point. 
Example 2. To illustrate the conceptual relation between priority swaps and changes in the order of precedence, we closely follow Example 1. The only difference is a small modification in the second case.

There are four schools $A=\{k, l, m, n\}$. Each school has two available slots. There are eight students $I=\left\{i_{1}, i_{2}, i_{3}, i_{4}, i_{5}, i_{6}, i_{7}, i_{8}\right\}$. There are two walk-zone students at each school: $I_{k}=$ $\left\{i_{1}, i_{2}\right\}, I_{l}=\left\{i_{3}, i_{4}\right\}, I_{m}=\left\{i_{5}, i_{6}\right\}$ and $I_{n}=\left\{i_{7}, i_{8}\right\}$. The random tiebreaker $\pi^{o}$ orders the students as:

$$
\pi^{o}: i_{1} \succ i_{8} \succ i_{3} \succ i_{4} \succ i_{5} \succ i_{6} \succ i_{7} \succ i_{2} .
$$

The preference profile is:

\begin{tabular}{cccccccc}
\hline$P^{i_{1}}$ & $P^{i_{2}}$ & $P^{i_{3}}$ & $P^{i_{4}}$ & $P^{i_{5}}$ & $P^{i_{6}}$ & $P^{i_{7}}$ & $P^{i_{8}}$ \\
\hline \hline$k$ & $k$ & $l$ & $l$ & $m$ & $m$ & $n$ & $k$ \\
$l$ & $l$ & $k$ & $k$ & $k$ & $k$ & $k$ & $l$ \\
$m$ & $m$ & $m$ & $m$ & $l$ & $l$ & $l$ & $m$ \\
$n$ & $n$ & $n$ & $n$ & $n$ & $n$ & $m$ & $n$ \\
\hline
\end{tabular}

First consider the case where each school has one walk-zone slot and one open slot. Also assume that, at each school, the walk-zone slot has higher precedence than the open slot.

The outcome of DA for this case is:

$$
\mu=\left(\begin{array}{cccccccc}
i_{1} & i_{2} & i_{3} & i_{4} & i_{5} & i_{6} & i_{7} & i_{8} \\
k & n & l & l & m & m & n & k
\end{array}\right) .
$$

Observe that six students (i.e. students $i_{1}, i_{3}, i_{4}, i_{5}, i_{6}, i_{7}$ ) are assigned to their walk-zone schools in this scenario.

Next, we change the order of precedence at school $k$ so that the open slot has higher precedence than the walk-zone slot. Each remaining school maintains the original order of precedence with the walk-zone slot higher precedence than the open slot.

The outcome of DA for the second case is:

$$
\mu^{\prime}=\left(\begin{array}{cccccccc}
i_{1} & i_{2} & i_{3} & i_{4} & i_{5} & i_{6} & i_{7} & i_{8} \\
k & k & l & m & m & n & n & l
\end{array}\right) .
$$

Observe that five students (i.e. students $i_{1}, i_{2}, i_{3}, i_{5}, i_{7}$ ) are assigned to their walk-zone schools in the second case. Thus, we see that the total number of walk-zone assignments decreases following reduction in the precedence of the walk-zone slot of school $k$.

\subsection{Additional Results for the Case of Two Schools}

In this section, we obtain sharper theoretical results by focusing on the case of two schools $(|A|=2)$. We assume that each student belongs to exactly one walk zone and that students rank both schools. This case is motivated in part by the commonly discussed policy objective of giving students from poorer neighborhoods access to desirable schools in richer neighborhoods. 
We have the following additional results in this more specialized setting

Proposition 3. Suppose that there are two schools, that each student belongs to exactly one walk zone, and that students rank both schools. Then, replacing an open slot of either school with a walk-zone slot weakly increases the total number of walk-zone assignments under DA.

An immediate implication of Proposition 3 is the following intuitive result justifying why the school choice and neighborhood schooling lobbies respectively prefer $0 \%$ and $100 \%$ walk-zone priority.

Corollary 1. Suppose that there are two schools, that each student belongs to exactly one walk zone, and that students rank both schools. Under DA (holding fixed the number of slots at each school):

- The minimum number of walk-zone assignments across all priority and precedence policies is obtained when all slots have open slot priority, and

- the maximum number of walk-zone assignments across all priority and precedence policies is obtained when all slots have walk-zone priority.

Proposition 4. Suppose that there are two schools, that each student belongs to exactly one walk zone, and that students rank both schools. Fix the set of walk-zone slots and the set of open slots at each school. Then, switching the order of precedence position of a walk-zone slot at either school with that of a subsequent open slot at that school weakly increases the total number of walk-zone assignments under DA.

Our empirical analysis in Section 3 shows that the fraction of students who receive their first choices, second choices, and so forth show virtually no response to changes in the fraction of walkzone slots or the order of precedence. Our final theoretical result provides a basis for this empirical observation.

Proposition 5. Suppose that there are two schools, that each student belongs to exactly one walk zone, and that students rank both schools. Then, the number of students assigned to their top-choice schools is independent of both the number of walk-zone slots and the choice of precedence order.

An important policy implication of our last result is that the division of slots between walkzone priority and open priority as well the order of precedence selection has little bearing on the aggregate number of students who receive their top choices; thus, the impact of these DA calibrations on student welfare is mostly distributional.

\section{Precedence and Priority in Boston Public Schools}

\section{$3.1 \quad 50-50$ vs. No Slot Split}

Examples 1 and 2 illustrate that even given priorities and precedence orders, determining how many students attend a walk-zone school is challenging without additional structure on priorities or preferences. These examples motivate the two-priority-type model presented in Section 2. 
To examine whether these comparative static predictions presented in our theoretical results capture the main features of school choice with richer priority structures, we use data on submitted preferences from Boston Public Schools. Relative to our two-priority-type model, Boston has three additional priority groups:

1. guaranteed applicants, who are typically continuing on at their current schools,

2. sibling-walk applicants, who have siblings currently attending a school and live in the walk zone, and

3. sibling applicants, who have siblings attending a school and live outside the walk zone. Under BPS's slot priorites, applicants are ordered as follows:

\begin{tabular}{c|c} 
Walk-Zone Slots & Open Slots \\
\hline Guaranteed & Guaranteed \\
Sibling-Walk & Sibling-Walk, Sibling \\
Sibling & \\
Walk & Walk, No Priority \\
No Priority &
\end{tabular}

A single random lottery number is used to order students within priority groups, and this number is the same for both types of slots.

We use data covering four years from 2009-2012, when BPS employed a mechanism based on the student-proposing deferred acceptance algorithm. Students interested in enrolling in or switching schools are asked to list schools each January for the first round. Students entering kindergarten can either apply for elementary school at Grade K1 or Grade K2 depending on whether they are four or five years old. Since the mechanism is based on the student-proposing deferred acceptance algorithm and there is no restriction on the number of schools that can be ranked, the assignment mechanism is strategy-proof. ${ }^{5}$ BPS informs families of this property on the application form, advising:

List your school choice in your true order of preference. If you list a popular school first, you won't hurt your chances of getting your second choice school if you don't get your first choice (BPS 2012).

Since the BPS mechanism is strategy-proof, we can isolate the effects of changes in priorities and precedence by holding submitted preferences fixed. ${ }^{6}$

\footnotetext{
${ }^{5}$ For analysis of the effects of restricting the number of choices which can be submitted, see Haeringer and Klijn (2009), Calsamiglia, Haeringer, and Kljin (2010), and Pathak and Sönmez (2013).

${ }^{6}$ As a check on our understanding of the data, we verify that we can recreate the assignments produced by BPS. Across four years and three applicant grades, we can match $98 \%$ of the assignments. Based on discussions with BPS, we learned that the reason why we do not exactly recreate the BPS assignment is that we do not have access to BPS's exact capacity file, and instead must construct it ex-post based on the final assignment. There are small differences between this measure of capacity and the capacity input to the algorithm due to the handling of unassigned students who are administratively assigned. In this paper, to hold this feature fixed in our counterfactuals, we take our re-creation as representing the BPS assignment.
} 
The motivating puzzle for this paper is shown in Table 1, which reports a comparison of the assignment produced by BPS, which relies on a 50-50 split of slots, to two extreme alternatives representing the ideal positions of the school choice and neighborhood school factions: (1) a priority structure without walk-zone priorities at any slot and (2) a priority structure where walk-zone priority applies at all slots. We refer to these two extremes as $\mathbf{0 \%}$ Walk and $\mathbf{1 0 0 \%}$ Walk, and we compute their outcomes using the same lottery numbers as BPS. Table 1 shows that the BPS assignment is nearly identical to the former of these two alternatives; it differs for only $3 \%$ of Grade K1 students. One might suspect that this phenomenon is driven by a strong preferences for neighborhood schools among applicants, which would bring the outcomes of these two assignment policies close together. However, comparing the $100 \%$ Walk outcome to BPS outcome, $19 \%$ of Grade K1 students obtain a different assignment. Therefore, the remarkable proximity of the current BPS outcome to the ideal of school choice proponents does not suggest (or reflect) negligible stakes in school choice.

For Grades K2 and 6, the BPS assignment is similarly close to the 0\% Walk outcome. Just as with Grade K1, this fact is not driven by applicants' preferences for neighborhood schools. The fraction of students who obtain a different assignment under the $100 \%$ Walk alternative are $17 \%$ and $10 \%$, respectively. The differences are smaller at higher grades because there are more continuing students who obtain guaranteed priority. On average, $4.5 \%$ of Grade K2 applicants have guaranteed priority at their first choice compared to $13 \%$ of Grade 6 students. Hence, despite the adoption of a seemingly neutral 50-50 split, Table 1 shows that the BPS outcome is closely similar to the $0 \%$ Walk outcome across different grades and years.

\subsection{The Impact of Precedence}

To understand the source of this puzzle, in Table 2 we report the fraction of students who obtain a slot in a school in their walk zone under different priority and precedence policies. Each year, when a student is unassigned in BPS, they are administratively assigned via an informal process conducted by the central enrollment office. These students are reported as unassigned in Table 2, even though many are likely to eventually obtain an assignment. Unassigned students are also the reason why the fraction of students who obtain a walk zone school is less than $50 \%$ even though most applicants at Grades K1 and K2 rank walk-zone schools as their first choices. Among those who are assigned, the BPS mechanism assigns $62.6 \%$ of Grade K1 and $58.2 \%$ of Grade K2 students to schools in their walk zones.

Before turning to variations in precedence, we start by comparing the fraction of students assigned to a walk zone school under the two priority extremes of $0 \%$ Walk and $100 \%$ Walk. Recall that in our model, Proposition 3 states that the number of students assigned to a walk zone school increases when the walk zone percentage increases. Table 2 shows this prediction borne out in Grade K1, K2, and 6, even though BPS's priority structure is more complicated than that studied in our model. Corollary 1 suggests that the fraction of students who obtain walk-zone assignments under the $0 \%$ Walk and 100\% Walk policies provides a benchmark for what can be obtained under 
variations of priority or precedence given student demand. For Grade K1, this range spans from $46.2 \%$ to $57.4 \%$ walk-zone assignment; the $11.2 \%$ interval represents the maximum difference in allocation attainable through changes in either priorities or precedence Grade K1.

The first alternative precedence we consider is Walk-Open, under which all walk-zone slots precede the open slots. (The actual BPS implementation is a slight variation on Walk-Open in which applicants with sibling priority and outside the walk zone apply to the open slots before applying to the walk-zone slots.) Focusing on Walk-Open provides useful intuition because as Table 2 shows the BPS system produces an outcome very close to Walk-Open. Under Walk-Open, if there are more walk-zone applicants than slots, then the pool of walk-zone applicants will be depleted by the time the open slots start being filled. For instance, suppose a school has 100 slots, and there are 100 walk-zone applicants and 100 non-walk-zone applicants. With the Walk-Open precedence order, 50 of the 100 walk-zone applicants will fill the 50 walk-zone slots. The remaining competition for open slots is between 50 walk-zone applicants and 100 non-walk-zone applicants. Since there are twice as many non-walk-zone applicants as walk-zone applicants in this residual pool, the non-walk-zone students stand to get more of the open slots. This processing bias partly explains why having walk-zone applicants first apply to walk-zone slots ends up disadvantaging them.

Next we consider the Open-Walk precedence order in which all applicants fill open slots before filling walk-zone slots. This represents the other end of precedence policy spectrum. Proposition 4 states that in our model, switching the order of precedence positions of walk-zone slots with those of subsequent open slots weakly increases the total number of walk-zone assignments. For each grade, Table 2 shows that this effect appears in the data. $54.8 \%$ of Grade K1 students are assigned to their walk-zone schools under Open-Walk, relative to $46.6 \%$ with Walk-Open. The $8.2 \%$ range, all holding fixed the 50-50 split, represents $73 \%$ of the range attainable from going from $0 \%$ Walk to $100 \%$ Walk. For Grade K2, the two extreme precedence policies cover $74 \%$ of the $9.3 \%$ range between the priority policy extremes. For Grade 6, the two extreme precedence policies cover $67 \%$ of the $5.4 \%$ range between the extremes. Thus, we see that decisions about precedence order have impacts on the assignment of magnitude comparable to decisions about priorities.

The difference between Walk-Open and Open-Walk represents the range of walk zone assignments that can arise from alternative precedence orders all within the 50-50 split. We turn to intermediate precedence policies still holding the 50-50 split fixed. The first alternative we examine attempts to mitigate the bias caused processing all of the slots of a particular type at once. The Rotating precedence order alternates between walk-zone and open slots. Under Rotating, the fraction of students who are assigned to schools in their walk zones increases by $2.5 \%$ relative to the Walk-Open precedence policy for Grade K1, but is still closer to Walk-Open than Open-Walk. The reason that Rotating is closer to Walk-Open is that alternating slots only partly undoes the processing bias, as we describe next. 


\subsection{Lottery Numbers}

The other ingredient which accounts for the effect of precedence policies are the lottery numbers of applicants. Recall the earlier example where a school has 100 slots, and there are 100 walkzone applicants and 100 non-walk-zone applicants. Under the Walk-Open precedence order, the remaining walk-zone applicants are out-numbered in the competition for the remaining open slots. They also come from a pool of applicants with adversely selected lottery numbers. Under WalkOpen, the walk-zone slots are filled by the 50 walk-zone applicants with the highest lottery numbers among walk-zone applicants. The competition for open slots is among the 50 walk-zone students with lowest lottery numbers and the 100 non-walk-zone students. When walk-zone applicants are considered for slots at the open slots, their adversely selected lottery numbers systematically place them behind applicants without walk-zone priority, leaving them unlikely to obtain open slots.

The random number bias - created by the precedence order - renders the outcomes under WalkOpen precedence very similar to the assignment that arises when all slots are open. In our example with 100 slots, with no walk zone priority, on average, 50 slots would be assigned to walk-zone applicants and 50 would be assigned to non-walk-zone applicants. Hence, there is no difference between a system without walk zone priority and the Walk-Open precedence. Even though the official BPS policy (following the School Committee's 1999 policy declaration) states that there should be open competition at the open slots, walk-zone applicants are systematically disadvantaged in the competition for those slots.

A single lottery number is also the reason why the Rotating precedence order is much closer to Walk-Open than Open-Walk in Table 2. With Rotating, the pool of walk-zone applicants with favorable lottery numbers is depleted after the first few slots are allocated, so the bias in lottery numbers among the pool of walk-zone applicants re-emerges after a few rounds of rotation. While Rotating tackles the processing bias, with one lottery number, it still retains the random number bias.

If the mechanism is constrained to have one lottery number, it may be possible to combat the random number bias via the precedence order. ${ }^{7}$ The Compromise precedence order first fills half of the walk-zone slots, then fills all the open slots, and then the fills the second half of the walk-zone slots. It attempts to even out the treatment of walk-zone applicants through changes in the order of slots. Initially, when the first few open slots are processed, the walk-zone applicant pool has adversely selected lottery numbers, but this bias becomes less important by the time the last open slots are processed. As a result, the fraction of applicants who attend a school for which they have walk-zone priority is close the midpoint between Walk-Open and Open-Walk. The results of this policy are shown in column (5) of Table 2. At Grade K1, Compromise assigns 50.7\% to a walk-zone school - exactly the mid-point between the Walk-Open (46.6\%) and Open-Walk (54.8\%) assignments.

When more than one lottery number can be used, there are additional possibilities. Table 3

\footnotetext{
${ }^{7}$ In the next section, we describe how BPS initially preferred this treatment, partly because of the difficulty involved in explaining a system with more than one lottery number.
} 
reports on alternative policies which use two lottery numbers in ordering applicants, one for the walk-zone slots and one for the open slots. The results reported in this table allow us to examine the processing and random number biases in isolation. Column (2) reports on the Walk-Open precedence order with two lottery numbers. This deals with the random number bias, but retains the processing bias, since the pool of applicants from the walk zone is still depleted by the time the open slots are filled. Walk-Open with two lottery numbers is still close to the current BPS outcome. It assigns $48.1 \%$ of students to walk-zone schools at Grade $\mathrm{K} 1$ and is quite close to the $46.6 \%$ assigned when Walk-Open is used with only one lottery number. Walk-Open with two lottery numbers is still closer to $0 \%$ Walk than $100 \%$ Walk; this suggests that random number bias accounts for only part of the reason the 50-50 allocation is not midway between the two extremes.

To deal with both the processing and random number bias, we investigate a Rotating treatment which uses two independent lottery numbers, one for walk-zone slots and the other for open slots. For Grade K1, 51.7\% of students are assigned to walk-zone schools under this treatment; this point is near the $51.8 \%$ midpoint between $0 \%$ Walk and 100\% Walk. Moreover, the difference between column (2) and (3) shows the magnitude of the processing bias. This difference is $3.6 \%$, while the difference due to random number bias (comparing column (2) in Table 2 and column (3) in Table 3 ) is $1.5 \%$. This suggests that both biases are substantial. The patterns are similar for Grades K2 and 6 .

The remedy of using two lottery numbers, however, has an important drawback. It is wellknown that using multiple lottery numbers across schools with deferred acceptance may generate efficiency losses (Abdulkadiroğlu, Pathak, and Roth 2009). Even though the two lottery numbers are within schools (and not across schools), the same efficiency consequence arises here. Indeed, if we compare the Unassigned row in Table 2 to Table 3, there are slightly more unassigned students when two lottery numbers are used. For Grade K1, 25.0\% of students are unassigned when using Rotating two lottery numbers, and this fraction is between $0.2-0.4 \%$ higher than any precedence policy reported in Table 2. The same pattern holds for Grade K2 and Grade 6. Though these are small numbers, they do suggest the possibility of inefficiency from two lottery numbers.

If the efficiency cost is prohibitive or explaining a system with two lottery numbers is too challenging, then the Open-Walk precedence order with a single lottery number is a viable alternative. By removing the statistical bias in the processing for walk-zone applicants, Open-Walk respects the school committee's goal of keeping the competition for non-walk seats open to all applicants. One implication of the Open-Walk treatment, we saw in Table 2, is that it leads to the highest fraction of students with a walk zone assignment within the 50-50 split. Therefore, adoption of Open-Walk may require calibration of the fraction of walk zone slots.

We report on such a calibration in Table 4, where we investigate what policy BPS has been implementing from 2009-2012. We compare BPS to the two alternatives we've proposed: OpenWalk with one lottery number and Rotating with two lottery numbers. Table 4 reports how the actual BPS implementation compares to the Open-Walk treatment with a smaller set of walk zone seats.

From 2009-2012, BPS's implementation corresponds to Open-Walk with a 5-10\% walk-zone 
priority depending on the grade. This fact stands in sharp contrast to the advertised 50-50 split. For Grade K1, the actual BPS implementation gives $47.2 \%$ of students walk-zone assignments; this is just above the Open-Walk treatment with $5 \%$ walk share, but below the Open-Walk treatment with $10 \%$ walk share. For Grade K2, the actual BPS implementation has $48.5 \%$ walk-zone assignment compared to $48.4 \%$ with the Open-Walk treatment with a $10 \%$ walk share. For Grade 6 , the actual BPS implementation is bracketed by Open-Walk with $5 \%$ and $10 \%$ walk share. BPS's implementation also corresponds to Rotating with two lottery numbers where the fraction of walk zone seats is just above $10 \%$.

Finally, the last issue we examine empirically is whether these changes are mostly distributional as suggested by Proposition 5. Table 5 reports on how the overall distribution of choices received varies with precedence order. This table shows that there is almost no difference in the aggregate distribution of received choice rank across BPS, rotating with two lottery numbers and Open-Walk with one lottery number. Consistent with Proposition 5, changes in precedence are a tool to achieve distributional objectives, having little overall impact on the total number of students who obtain their top choices.

\section{The 2012-2013 Boston Debate between Neighborhood and Choice}

The debate between neighborhood and choice proponents was rekindled in dramatic fashion in Boston following Mayor Thomas Menino's 2012 State of the City Address. ${ }^{8}$ In the speech, Menino (2012) articulated support for the faction in favor of greater neighborhood assignment:

"Something stands in the way of taking our [public school] system to the next level: a student assignment process that ships our kids to schools across our city. Pick any street. A dozen children probably attend a dozen different schools. Parents might not know each other; children might not play together. They can't carpool, or study for the same tests. $[\ldots]$

Boston will have a radically different school assignment process-one that puts priority on children attending schools closer to their homes."

For elementary and middle school admissions, Boston is currently divided into three zones: North, West, and East. To respond to Menino's charge, a natural proposal is to try to increase walk-zone assignment by increasing the proportion of walk-zone slots at each school. But no one knew at the time of Menino's speech that the current system was unintentionally disadvantaging walk-zone students in the competition for open slots.

In Fall 2012, BPS proposed five different plans which all restricted participant choice by reducing the number of schools students could rank. ${ }^{9}$ The idea behind each of these plans was to reduce competition from non-neighborhood applicants at the open half of each school. These plans and

\footnotetext{
${ }^{8}$ For more on this debate, see the materials available at http://bostonschoolchoice.org and press accounts by Goldstein (2012) and Handy (2012).

${ }^{9}$ The initial plans suggested dividing the city into $6,9,11$, or 23 zones, or assignment based purely on neighborhood.
} 
other proposals from the community became the center of a year-long, city-wide discussion on school choice. Critics expressed concerns that with smaller choice menus, families from disadvantaged neighborhoods would be shut out of good schools if the neighborhood component of assignment were given more weight. This point was summarized by a community activist (Seelye 2012):

"A plan that limits choice and that is strictly neighborhood-based gets us to a system that is more segregated than [BPS] is now."

Underlying the discussion was puzzlement as to how Menino's concerns could be correct given that a walk zone applicant always obtains a seat ahead of a non-walk zone applicant at $50 \%$ of school seats and remaining walk zone applicants have a shot at the open half. A few members of the neighborhood assignment faction were convinced that there had not been enough neighborhood assignment in recent years, but they could not determine why. After a preliminary version of our research became available, Pathak and Sönmez interacted with BPS's staff. Parts of our research were presented to the Mayor's twenty-seven-member Executive Advisory Committee (EAC), where it became known that the BPS walk-zone priority was not having its intended impact because of the chosen precedence order. The EAC meeting minutes summarized the discussion (EAC 2013):

"A committee member stated that the walk-zone priority in its current application does not have a significant impact on student assignment. The committee member noted that this finding was consistent with anecdotal evidence that the committee had heard from parents."

Following the presentation, BPS immediately recommended that the system switch to the compromise treatment for the Fall 2013 admissions cycle. The meeting minutes state:

"BPS's recommendation is to utilize the compromise method in order to ensure that the walk-zone priority is not causing an unintended consequence that is not in stated policy."

Part of the reason for recommending the Compromise method is the anticipated difficulty of describing a system employing two lottery numbers.

The switch to the Compromise treatment, as Table 2 shows, leads to an increase in the number of students assigned to their walk-zone schools. This change, together with the proposals to shrink zones or adopt a plan with a smaller choice menus, raised concerns that the equity of access would decrease. Our discovery about the role of precedence proved so significant that it became part of the fight between those favoring neighborhood assignment and those favoring increased choice.

On one hand, proponents of neighborhood assignment interpreted our findings as showing that the unintentional improper implementation of the 50-50 school split caused hundreds of students to be shut out of their neighborhood schools. They argued that a change in the precedence order would be the only policy consistent with the School Committee's policy in 1999. Moving either to Rotating with two lottery numbers or Open-Walk would also coincide with the Mayor's push towards moving children closer to home.

Choice proponents seized on our findings for two distinct points. First, some groups, such as the activist Metropolitan Area Planning Council, fought fiercely to maintain to keep the 50-50 seat split with the current precedence order (MAPC 2013): 
"The assignment priority given to walk-zone students has profound impacts on the outcomes of any new plan. The possible changes that have been proposed or discussed include increasing the set-aside, decreasing the set-aside, changing the processing order, or even reducing the allowable distance for walk zone priority to less than a mile. Actions that provide additional advantage to walk-zone students are likely to have a disproportionate negative impact on Black and Hispanic students, who are more reliant on out-of-walk-zone options for the quality schools in their basket."

The symbolism of the 50-50 split resounded with sophisticated choice proponents because it created the impression that they were giving something away to neighborhood proponents even though they really were not due to the BPS implementation.

Some other parties refused to let go of the idea that walk-zone priority as currently implemented did not have large implications for the assignment. For instance, the City Councillor in charge of education publicly testified (Connolly 2013):

"MIT tells us that so many children in the walk zones of high demand schools 'flood the pool' of applicants, and that children in these walk zones get in in higher numbers, so walk zone priority doesn't really matter."

"Maybe, that is true. But if removing the walk zone priority doesn't change anything, why change it all?"

Choice proponents also interpreted our findings as an argument for removing walk-zone priority entirely. Indeed, given that walk-zone priority plays a relatively small role (as currently implemented by BPS relative to $0 \%$ Walk), simply eliminating it might increase transparency about how the system works. Getting rid of walk zone priority altogether avoids the (false) impression that applicants from the walk zone are receiving a boost under the mechanism.

This argument eventually convinced Boston Superintendent Carol Johnson to overturn the Mayor's committee's recommendation to keep the existing processing order. On March 13, 2013, Superintendent Johnson stated (Johnson 2013):

"After viewing the final MIT and BC presentations on the way the walk zone priority actually works, it seems to me that it would be unwise to add a second priority to the Home-Based model by allowing the walk zone priority be carried over."

. .

"Leaving the walk zone priority to continue as it currently operates is not a good option. We know from research that it does not make a significant difference the way it is applied today: although people may have thought that it did, the walk zone priority does not in fact actually help students attend schools closer to home. The External Advisory Committee suggested taking this important issue up in two years, but I believe we are ready to take this step now. We must ensure the Home-Based system works in an honest and transparent way from the very beginning." 
In March 2013, BPS voted to totally eliminate walk zone priority, so as to avoid giving the false impression of advantaging walk-zone students. The district also voted for a Home-Based system which defines the choice menu for applicants based on their address, and substantially reduces the number of schools applicants can rank. This change affects applicants for elementary and middle school starting Fall 2013, but not those for Grade 9, where as of this draft, admissions are city-wide and still retain the 50-50 split with the existing BPS precedence order.

\section{Conclusion}

Those articulating a pro-neighborhood position in the school choice debate often lament how choice has "destroyed the concept of neighborhood schools" by scattering children across the city by assigning them to schools far from home (Ravitch 2011). In Boston, Mayor Menino's claim that the current system does not put priority on children attending schools closer to their homes seemed to be at odds with the fact that half of each school's seats prioritized applicants from the walk-zone. This paper explains this apparent puzzle by showing the important role played by the precedence order in the deferred acceptance algorithm.

In addition to the two new comparative statics results, the paper has shown how the precedence order effectively undermined the policy aim of the 50-50 slot split in Boston. Moreover, our empirical results show that in Boston, the precedence order (1) has quantitative impacts almost as large as changes in neighborhood priority, and (2) is an important lever for achieving distributional objectives. The role precedence played was so central in Boston that once its unintended consequences were made transparent, policymakers decided to abandon walk zone priority altogether.

Even though explicit implementation of precedence has not been part of prior school choice policy discussions (with the exception of the recent one at BPS), it is clear that they should accompany debates about priorities. It also seems likely that precedence could play an important role outside of student assignment, in other priority-based assignment problems where priorities depend on particular slots. Finally, it is worth noting that our paper uses market design techniques and analysis to show how to achieve given policy objectives. We have not considered normative questions like whether there should be walk-zone priority at all, or how to compute the optimal walk-zone set aside. These important questions seem worth future investigation. 


\section{A Appendix}

\section{A.1 Preliminaries for Proposition 1}

For a school $a^{*}$ and a slot $s^{*} \in S^{a^{*}}$ of school $a^{*}$, suppose that $s^{*}$ is an open slot under priority structure $\pi$, and is a walk-zone slot under priority $\tilde{\pi}$. Suppose furthermore that $\pi^{s}=\tilde{\pi}^{s}$ for all slots $s \in S^{a^{*}}$ other than $a^{*}$. Let $C^{a^{*}}$ and $\tilde{C}^{a^{*}}$ respectively be the choice functions for $a^{*}$ induced by the priorities $\pi$ and $\tilde{\pi}$, under (fixed) precedence order $\triangleright^{a^{*}}$.

Lemma 1. For any set of students $\bar{I} \subseteq I$ :

1. All students in the walk-zone of $a^{*}$ that are chosen from $\bar{I}$ under choice function $C^{a^{*}}$ are chosen under choice function $\tilde{C}^{a^{*}}\left(\right.$ i.e. $\left.\left[\left(C^{a^{*}}(\bar{I})\right) \cap I_{a^{*}}\right] \subseteq\left[\left(\tilde{C}^{a^{*}}(\bar{I})\right) \cap I_{a^{*}}\right]\right)$.

2. All students not in the walk-zone of $a^{*}$ that are from $\bar{I}$ chosen under choice function $\tilde{C}^{a^{*}}$ are chosen under choice function $C^{a^{*}}\left(\right.$ i.e. $\left.\left[\left(\tilde{C}^{a^{*}}(\bar{I})\right) \cap\left(I \backslash I_{a^{*}}\right)\right] \subseteq\left[\left(C^{a^{*}}(\bar{I})\right) \cap\left(I \backslash I_{a^{*}}\right)\right]\right)$.

Proof. We proceed by induction on the number $q_{a^{*}}$ of slots at $a^{*}$. The base case $q_{a^{*}}=1$ is immediate, as then $S^{a^{*}}=\left\{s^{*}\right\}$ and $C^{a^{*}}(\bar{I}) \neq \tilde{C}^{a^{*}}(\bar{I})$ if and only if a walk-zone student of $a^{*}$ is assigned to $s^{*}$ under $\tilde{C}$, but a non-walk-zone student is assigned to $s^{*}$ under $C$, that is, if $\tilde{C}^{a^{*}}(\bar{I}) \subseteq I_{a^{*}}$ while $C^{a^{*}}(\bar{I}) \subseteq I \backslash I_{a^{*}}$. It follows immediately from this observation that $\left[\left(C^{a^{*}}(\bar{I})\right) \cap\right.$ $\left.I_{a^{*}}\right] \subseteq\left[\left(\tilde{C}^{a^{*}}(\bar{I})\right) \cap I_{a^{*}}\right]$ and $\left[\left(\tilde{C}^{a^{*}}(\bar{I})\right) \cap\left(I \backslash I_{a^{*}}\right)\right] \subseteq\left[\left(C^{a^{*}}(\bar{I})\right) \cap\left(I \backslash I_{a^{*}}\right)\right]$.

Now, given the result for the base case $q_{a^{*}}=1$, we suppose that the result holds for all $q_{a^{*}}<\ell$ for some $\ell \geq 1$; we show that this implies the result for $q_{a^{*}}=\ell$. We suppose that $q_{a^{*}}=\ell$, and let $\bar{s} \in S^{a^{*}}$ be the slot which is minimal (i.e., processed last) under the precedence order $\triangleright^{a^{*}}$. A student eligible for one type of slot is also eligible for the other, and hence $\bar{s}$ is either full in both cases or empty in both cases. Moreover, the result follows directly from the inductive hypothesis in the case if no student is assigned to $\bar{s}$ (under either priority structure); hence, we assume that

$$
\left|C^{a^{*}}(\bar{I})\right|=\left|\tilde{C}^{a^{*}}(\bar{I})\right|=q_{a^{*}}=\ell .
$$

If $\bar{s}=s^{*}$, then the result follows just as in the base case: It is clear from the algorithms defining $C^{a^{*}}$ and $\tilde{C}^{a^{*}}$ that the same students are assigned to slots $s \triangleright^{a^{*}} s^{*}=\bar{s}$ in the computations of $C^{a^{*}}(\bar{I})$ and $\tilde{C}^{a^{*}}(\bar{I})$, as those slots' priorities and relative precedence ordering fixed. Thus, as in the base case, $C^{a^{*}}(\bar{I}) \neq \tilde{C}^{a^{*}}(\bar{I})$ if and only if a walk-zone student of $a^{*}$ is assigned to $s^{*}$ under $\tilde{C}$, but a non-walk-zone student is assigned to $s^{*}$ under $C$.

If $\bar{s} \neq s^{*}$, then $s^{*} \triangleright^{a^{*}} \bar{s}$. We let $J \subseteq \bar{I}$ be the set of students assigned to slots in $S^{a^{*}} \backslash\{\bar{s}\}$ in the computation of $C^{a^{*}}(\bar{I})$, and let $\tilde{J} \subseteq \bar{I}$ be the set of students assigned to slots in $S^{a^{*}} \backslash\{\bar{s}\}$ in the computation of $\tilde{C}^{a^{*}}(\bar{I})$. The inductive hypothesis, in the case $q_{a^{*}}=\ell-1$, implies

$$
\begin{aligned}
{\left[J \cap I_{a^{*}}\right] } & \subseteq\left[\tilde{J} \cap I_{a^{*}}\right], \\
{\left[\tilde{J} \cap\left(I \backslash I_{a^{*}}\right)\right] } & \subseteq\left[J \cap\left(I \backslash I_{a^{*}}\right)\right],
\end{aligned}
$$

as the first $q_{a^{*}}$ slots of $a^{*}$ can be treated as a school with slot-set $S^{a^{*}} \backslash\{\bar{s}\}$ (under the precedence order induced by $\left.\triangleright^{a^{*}}\right)$. 
If we have equality in (2) and (3), ${ }^{10}$ then the set of students available to be assigned to $\bar{s}$ in the computation of $C^{a^{*}}(\bar{I})$ is the same as in the computation of $\tilde{C}^{a^{*}}(\bar{I})$. Thus, since $\pi^{\bar{s}}=\tilde{\pi}^{\bar{s}}$ by assumption, we have $C^{a^{*}}(\bar{I})=\tilde{C}^{a^{*}}(\bar{I})$; hence, the desired result follows trivially.

If instead the inclusions in (2) and (3) are strict, then there is some student $i \in\left[\tilde{J} \cap I_{a^{*}}\right] \backslash\left[J \cap I_{a^{*}}\right]$ who is in the walk-zone of $a^{*}$ and is assigned to a slot $s \triangleright^{a^{*}} \bar{s}$ in the computation of $\tilde{C}^{a^{*}}(\bar{I})$ but is not assigned to such a slot in the computation of $C^{a^{*}}(\bar{I})$. We let $\bar{i}$ be the student in $\left[\tilde{J} \cap I_{a^{*}}\right] \backslash\left[J \cap I_{a^{*}}\right]$ ranked highest under $\pi^{o}$; by construction, $\bar{i}$ must be the $\pi^{o}$-maximal student in $[\bar{I} \backslash J] \cap I_{a^{*}}$. Thus:

- If $\bar{s}$ is assigned a walk-zone student of $a^{*}$ in the computation of $C^{a^{*}}(\bar{I})$, then that student must be $\bar{i}$. Then, $C^{a^{*}}(\bar{I})=J \cup\{\bar{i}\}$; hence,

$$
\left[\left(C^{a^{*}}(\bar{I})\right) \cap I_{a^{*}}\right]=\left[(J \cup\{\bar{i}\}) \cap I_{a^{*}}\right] \subseteq\left[\tilde{J} \cap I_{a^{*}}\right] \cup\{\bar{i}\},
$$

where the inequality follows from (2). Since $\bar{i} \in\left[\tilde{J} \cap I_{a^{*}}\right] \subseteq\left[\left(\tilde{C}^{a^{*}}(\bar{I})\right) \cap I_{a^{*}}\right]$, it follows that

$$
\left[\left(C^{a^{*}}(\bar{I})\right) \cap I_{a^{*}}\right] \subseteq\left[\left(\tilde{C}^{a^{*}}(\bar{I})\right) \cap I_{a^{*}}\right] .
$$

- If $\bar{s}$ is assigned a student not in the walk-zone of $a^{*}$ in the computation of $C^{a^{*}}(\bar{I})$, then $(2)$ directly implies (4).

This completes the first half of the induction.

Likewise, if the inclusions in (2) and (3) are strict, then there is some student $i \in\left[J \cap\left(I \backslash I_{a^{*}}\right)\right] \backslash$ $\left[\tilde{J} \cap\left(I \backslash I_{a^{*}}\right)\right]$ who is not in the walk-zone of $a^{*}$, is assigned to a slot $s \triangleright^{a^{*}} \bar{s}$ in the computation of $C^{a^{*}}(\bar{I})$, and is not assigned to such a slot in the computation of $\tilde{C}^{a^{*}}(\bar{I})$. We let $\hat{i}$ be the student in $\left[J \cap\left(I \backslash I_{a^{*}}\right)\right] \backslash\left[\tilde{J} \cap\left(I \backslash I_{a^{*}}\right)\right]$ ranked highest under $\pi^{o}$; by construction, $\hat{i}$ must be the $\pi^{o}$-maximal student in $[\bar{I} \backslash \tilde{J}] \cap\left(I \backslash I_{a^{*}}\right)$. Thus:

- If $\bar{s}$ is assigned a student not in the walk zone of $a^{*}$ in the computation of $\tilde{C}^{a^{*}}(\bar{I})$, then that student must be $\hat{i}$. Then, $\tilde{C}^{a^{*}}(\bar{I})=\tilde{J} \cup\{\hat{i}\}$; hence,

$$
\left[\left(\tilde{C}^{a^{*}}(\bar{I})\right) \cap\left(I \backslash I_{a^{*}}\right)\right]=\left[(\tilde{J} \cup\{\hat{i}\}) \cap\left(I \backslash I_{a^{*}}\right)\right] \subseteq\left[J \cap\left(I \backslash I_{a^{*}}\right)\right] \cup\{\hat{i}\},
$$

where the inequality follows from (3). Since $\hat{i} \in\left[J \cap\left(I \backslash I_{a^{*}}\right)\right] \subseteq\left[\left(C^{a^{*}}(\bar{I})\right) \cap\left(I \backslash I_{a^{*}}\right)\right]$, it follows that

$$
\left[\left(\tilde{C}^{a^{*}}(\bar{I})\right) \cap\left(I \backslash I_{a^{*}}\right)\right] \subseteq\left[\left(C^{a^{*}}(\bar{I})\right) \cap\left(I \backslash I_{a^{*}}\right)\right] .
$$

- If $\bar{s}$ is assigned a walk-zone student of $a^{*}$ in the computation of $\tilde{C}^{a^{*}}(\bar{I})$, then (3) directly implies (5).

These observations complete the second half of the induction.

\footnotetext{
${ }^{10} \mathrm{As}|J|=|\tilde{J}|$ by (1), equality holds in one of (2) and (3) if and only if it holds for both inclusions (2) and (3).
} 


\section{A.2 Preliminaries for Proposition 2}

For a school $a^{*}$ and a slot $s_{w}^{*} \in S^{a^{*}}$ of school $a^{*}$, suppose that $s_{w}^{*}$ is a walk-zone slot. Suppose that precedence order $\tilde{\nabla}$ is obtained from $\triangleright$ by swapping the positions of $s_{w}^{*}$ and some open slot $s_{o}^{*} \in S^{a^{*}}$ that is below $s_{w}^{*}$ in the order $\triangleright^{a^{*}}$ (i.e. $s_{w}^{*} \triangleright^{a^{*}} s_{o}^{*}$ ), and leaving the positions of all other slots unchanged. Let $C^{a^{*}}$ and $\tilde{C}^{a^{*}}$ respectively be the choice functions for $a^{*}$ induced by the precedence orders $\triangleright$ and $\tilde{\triangleright}$, under (fixed) slot priorities $\pi^{s}\left(s \in S^{a^{*}}\right)$.

Lemma 2. For any set of students $\bar{I} \subseteq I$ :

1. All students in the walk-zone of $a^{*}$ that are chosen from $\bar{I}$ under choice function $C^{a^{*}}$ are chosen under choice function $\tilde{C}^{a^{*}}$ (i.e. $\left.\left[\left(C^{a^{*}}(\bar{I})\right) \cap I_{a^{*}}\right] \subseteq\left[\left(\tilde{C}^{a^{*}}(\bar{I})\right) \cap I_{a^{*}}\right]\right)$.

2. All students not in the walk-zone of $a^{*}$ that are from $\bar{I}$ chosen under choice function $\tilde{C}^{a^{*}}$ are chosen under choice function $C^{a^{*}}$ (i.e. $\left.\left[\left(\tilde{C}^{a^{*}}(\bar{I})\right) \cap\left(I \backslash I_{a^{*}}\right)\right] \subseteq\left[\left(C^{a^{*}}(\bar{I})\right) \cap\left(I \backslash I_{a^{*}}\right)\right]\right)$.

Proof. We proceed by induction on the number $q_{a^{*}}$ of slots at $a^{*}$.

First, we prove the base case $q_{a^{*}}=2 .{ }^{11}$ We denote by $i_{s_{w}^{*}}$ and $i_{s_{o}^{*}}$ (resp. $\tilde{i}_{s_{w}^{*}}$ and $\tilde{i}_{s_{o}^{*}}$ ) the students respectively assigned to slots $s_{w}^{*}$ and $s_{o}^{*}$ in the computation of $C^{a^{*}}(\bar{I})\left(\operatorname{resp} . \tilde{C}^{a^{*}}(\bar{I})\right)$. Now:

- If $\left\{i_{s_{w}^{*}}, i_{s_{o}^{*}}\right\} \subset I_{a^{*}}$, then the ordering under $\pi^{o}$ must take the form

$$
\pi^{o}: i_{s_{w}^{*}} \succ i_{s_{o}^{*}} \succ \cdots
$$

as otherwise some student $i \neq i_{s_{w}^{*}}$ would have higher rank than $i_{s_{o}^{*}}$ under $\pi^{o}$, and would thus have higher claim than $i_{s_{o}^{*}}$ for (open) slot $s_{o}^{*}$ under precedence order $\triangleright^{a^{*}}$. But then, $i_{s_{w}^{*}}$ is the $\pi^{o}$-maximal student in $\bar{I}$ and $i_{s_{o}^{*}}$ is the $\pi^{o}$-maximal walk-zone student in $\bar{I} \backslash\left\{i_{s_{w}^{*}}\right\}$; hence, we must have $\tilde{i}_{s_{o}^{*}}=i_{s_{w}^{*}}$ and $\tilde{i}_{s_{w}^{*}}=i_{s_{o}^{*}}$, so that $\tilde{C}^{a^{*}}(\bar{I})=C^{a^{*}}(\bar{I})$.

- If $\left\{i_{s_{w}^{*}}, i_{s_{o}^{*}}\right\} \subset\left(I \backslash I_{a^{*}}\right)$, then $\bar{I}$ contains no students in the walk-zone of $a^{*}$ (i.e. $\left.\bar{I} \cap I_{a^{*}}=\emptyset\right)$ and $i_{s_{w}^{*}}$ and $i_{s_{o}^{*}}$ are then just the $\pi^{o}$-maximal non-walk-zone students in $\bar{I}$. In this case, we find that $\tilde{i}_{s_{o}^{*}}=i_{s_{w}^{*}}$ and $\tilde{i}_{s_{w}^{*}}=i_{s_{o}^{*}}$; hence, $\tilde{C}^{a^{*}}(\bar{I})=C^{a^{*}}(\bar{I})$.

- If $i_{s_{w}^{*}} \in I_{a^{*}}$ and $i_{s_{o}^{*}} \in\left(I \backslash I_{a^{*}}\right)$, then $i_{s_{w}^{*}}$ is the $\pi^{o}$-maximal walk-zone student of $a^{*}$ in $\bar{I}$. If $i_{s_{w}^{*}}$ is also $\pi^{o}$-maximal among all students in $\bar{I}$, then we have $\tilde{i}_{s_{o}^{*}}=i_{s_{w}^{*}}$. Moreover, in this case either $\tilde{i}_{s_{w}^{*}} \in I_{a^{*}}$, or $\tilde{i}_{s_{w}^{*}}$ is the only walk-zone student of $a^{*}$ in $\bar{I}$, so that $\tilde{i}_{s_{w}^{*}}=i_{s_{o}^{*}}$.

Alternatively, if $i_{s_{w}^{*}}$ is not $\pi^{o}$-maximal among all students in $\bar{I}$, then $i_{s_{o}^{*}}$ must be $\pi^{o}$-maximal among all students in $\bar{I}$, so that $\tilde{i}_{s_{o}^{*}}=i_{s_{o}^{*}}$ and $\tilde{i}_{s_{w}^{*}}=i_{s_{w}^{*}}$. In either case, we have $\left[\left(C^{a^{*}}(\bar{I})\right) \cap\right.$ $\left.I_{a^{*}}\right]=\left\{i_{s_{w}^{*}}\right\} \subseteq\left[\left(\tilde{C}^{a^{*}}(\bar{I})\right) \cap I_{a^{*}}\right]$. Additionally, we have $\left[\left(\tilde{C}^{a^{*}}(\bar{I})\right) \cap\left(I \backslash I_{a^{*}}\right)\right] \subseteq\left\{i_{s_{o}^{*}}\right\}=\left[\left(C^{a^{*}}(\bar{I})\right) \cap\right.$ $\left.\left(I \backslash I_{a^{*}}\right)\right]$.

- We cannot have $i_{s_{w}^{*}} \in\left(I \backslash I_{a^{*}}\right)$ and $i_{s_{o}^{*}} \in I_{a^{*}}$, as $s_{w}^{*}$ is a walk-zone slot (and thus gives all students in $I_{a^{*}}$ higher priority than students in $\left.I \backslash I_{a^{*}}\right)$ and $s_{w}^{*} \triangleright^{a^{*}} s_{o}^{*}$.

\footnotetext{
${ }^{11}$ Note that the setup requires at least two distinct slots of $a^{*}$, so $q_{a^{*}}=2$ a priori.
} 
The preceding four cases are exhaustive and the desired result holds in each; thus, we have the base case.

Now, given the result for the base case $q_{a^{*}}=2$, we suppose that the result holds for all $q_{a^{*}}<\ell$ for some $\ell \geq 2$; we show that this implies the result for $q_{a^{*}}=\ell$. We observe that it suffices to show the result in the case that $s_{w}^{*}$ and $s_{o}^{*}$ are adjacent under $\triangleright^{a^{*}}$; the result for general positions with $s_{w}^{*} \triangleright^{a^{*}} s_{o}^{*}$ follows from the adjacency case upon a sequence of adjacent-slot swaps. Thus, we suppose that $s_{w}^{*}$ and $s_{o}^{*}$ are adjacent under $\triangleright^{a^{*}}$, with $s_{w}^{*} \triangleright^{a^{*}} s_{o}^{*}$, and suppose that $q_{a^{*}}=\ell$. We let $\bar{s} \in S^{a^{*}}$ be the slot which is minimal under the precedence order $\triangleright^{a^{*}}$. A student eligible for one type of slot is also eligible for the other, and hence $\bar{s}$ is either full in both cases or empty in both cases. Moreover, the result follows directly from the inductive hypothesis in the case if no student is assigned to $\bar{s}$ (under either priority structure); hence, we assume that

$$
\left|C^{a^{*}}(\bar{I})\right|=\left|\tilde{C}^{a^{*}}(\bar{I})\right|=q_{a^{*}}=\ell .
$$

If $\bar{s}=s_{o}^{*}$, then the result follows just as in the base case, as it is clear from the algorithms defining $C^{a^{*}}$ and $\tilde{C}^{a^{*}}$ that the same students are assigned to slots $s \triangleright^{a^{*}} s_{w}^{*} \triangleright^{a^{*}} s_{o}^{*}=\bar{s}$ in the computations of $C^{a^{*}}(\bar{I})$ and $\tilde{C}^{a^{*}}(\bar{I})$.

If $\bar{s} \neq s_{o}^{*}$, then $s_{w}^{*} \triangleright^{a^{*}} s_{o}^{*} \triangleright^{a^{*}} \bar{s}$. We let $J \subseteq \bar{I}$ be the set of students assigned to slots in $S^{a^{*}} \backslash\{\bar{s}\}$ in the computation of $C^{a^{*}}(\bar{I})$, and let $\tilde{J} \subseteq \bar{I}$ be the set of students assigned to slots in $S^{a^{*}} \backslash\{\bar{s}\}$ in the computation of $\tilde{C}^{a^{*}}(\bar{I})$. The inductive hypothesis, in the case $q_{a^{*}}=\ell-1$, implies

$$
\begin{aligned}
{\left[J \cap I_{a^{*}}\right] } & \subseteq\left[\tilde{J} \cap I_{a^{*}}\right], \\
{\left[\tilde{J} \cap\left(I \backslash I_{a^{*}}\right)\right] } & \subseteq\left[J \cap\left(I \backslash I_{a^{*}}\right)\right],
\end{aligned}
$$

as the first $q_{a^{*}}$ slots of $a^{*}$ can be treated as a school with slot-set $S^{a^{*}} \backslash\{\bar{s}\}$ (under the precedence order induced by $\triangleright^{a^{*}}$ ).

If we have equality in (7) and (8), ${ }^{12}$ then the set of students available to be assigned to $\bar{s}$ in the computation of $C^{a^{*}}(\bar{I})$ is the same as in the computation of $\tilde{C}^{a^{*}}(\bar{I})$; the desired result then follows trivially.

If instead the inclusions in (7) and (8) are strict, then there is some student $i \in\left[\tilde{J} \cap I_{a^{*}}\right] \backslash\left[J \cap I_{a^{*}}\right]$ who is in the walk-zone of $a^{*}$ and is assigned to a slot $s \triangleright^{a^{*}} \bar{s}$ in the computation of $\tilde{C}^{a^{*}}(\bar{I})$ but is not assigned to such a slot in the computation of $C^{a^{*}}(\bar{I})$. We let $\bar{i}$ be the student in $\left[\tilde{J} \cap I_{a^{*}}\right] \backslash\left[J \cap I_{a^{*}}\right]$ ranked highest under $\pi^{o}$; by construction, $\bar{i}$ must be the $\pi^{o}$-maximal student in $[\bar{I} \backslash J] \cap I_{a^{*}}$. Thus:

- If $\bar{s}$ is assigned a walk-zone student of $a^{*}$ in the computation of $C^{a^{*}}(\bar{I})$, then that student must be $\bar{i}$. Then, $C^{a^{*}}(\bar{I})=J \cup\{\bar{i}\}$; hence,

$$
\left[\left(C^{a^{*}}(\bar{I})\right) \cap I_{a^{*}}\right]=\left[(J \cup\{\bar{i}\}) \cap I_{a^{*}}\right] \subseteq\left[\tilde{J} \cap I_{a^{*}}\right] \cup\{\bar{i}\},
$$

where the inequality follows from (7). Since $\bar{i} \in\left[\tilde{J} \cap I_{a^{*}}\right] \subseteq\left[\left(\tilde{C}^{a^{*}}(\bar{I})\right) \cap I_{a^{*}}\right]$, it follows that

$$
\left[\left(C^{a^{*}}(\bar{I})\right) \cap I_{a^{*}}\right] \subseteq\left[\left(\tilde{C}^{a^{*}}(\bar{I})\right) \cap I_{a^{*}}\right] .
$$

\footnotetext{
${ }^{12}$ As $|J|=|\tilde{J}|$ by (6), equality holds in one of (7) or (8) if and only if it holds for both (7) and (8).
} 
- If $\bar{s}$ is assigned a student not in the walk-zone of $a^{*}$ in the computation of $C^{a^{*}}(\bar{I})$, then $(7)$ directly implies (9).

Thus, we have completed the first half of the induction.

Likewise, if the inclusions in (7) and (8) are strict, then there is some student $i \in\left[J \cap\left(I \backslash I_{a^{*}}\right)\right] \backslash$ $\left[\tilde{J} \cap\left(I \backslash I_{a^{*}}\right)\right]$ who is not in the walk-zone of $a^{*}$, is assigned to a slot $s \triangleright^{a^{*}} \bar{s}$ in the computation of $C^{a^{*}}(\bar{I})$, and is not assigned to such a slot in the computation of $\tilde{C}^{a^{*}}(\bar{I})$. We let $\hat{i}$ be the student in $\left[J \cap\left(I \backslash I_{a^{*}}\right)\right] \backslash\left[\tilde{J} \cap\left(I \backslash I_{a^{*}}\right)\right]$ ranked highest under $\pi^{o}$; by construction, $\hat{i}$ must be the $\pi^{o}$-maximal student in $[\bar{I} \backslash \tilde{J}] \cap\left(I \backslash I_{a^{*}}\right)$. Thus:

- If $\bar{s}$ is assigned a student not in the walk zone of $a^{*}$ in the computation of $\tilde{C}^{a^{*}}(\bar{I})$, then that student must be $\hat{i}$. Then, $\tilde{C}^{a^{*}}(\bar{I})=\tilde{J} \cup\{\hat{i}\}$; hence,

$$
\left[\left(\tilde{C}^{a^{*}}(\bar{I})\right) \cap\left(I \backslash I_{a^{*}}\right)\right]=\left[(\tilde{J} \cup\{\hat{i}\}) \cap\left(I \backslash I_{a^{*}}\right)\right] \subseteq\left[J \cap\left(I \backslash I_{a^{*}}\right)\right] \cup\{\hat{i}\},
$$

where the inequality follows from (8). Since $\hat{i} \in\left[J \cap\left(I \backslash I_{a^{*}}\right)\right] \subseteq\left[\left(C^{a^{*}}(\bar{I})\right) \cap\left(I \backslash I_{a^{*}}\right)\right]$, it follows that

$$
\left[\left(\tilde{C}^{a^{*}}(\bar{I})\right) \cap\left(I \backslash I_{a^{*}}\right)\right] \subseteq\left[\left(C^{a^{*}}(\bar{I})\right) \cap\left(I \backslash I_{a^{*}}\right)\right] .
$$

- If $\bar{s}$ is assigned a walk-zone student of $a^{*}$ in the computation of $C^{a^{*}}(\bar{I})$, then (8) directly implies (10).

These observations complete the second half of the induction.

\section{A.3 Proof of Propositions 1 and 2}

Definition. In the cumulative offer process under choice functions $\bar{C}$, students to schools in a sequence of steps $\ell=1,2, \ldots$ :

Step 1. Some student $i^{1} \in I$ proposes to his favorite school $a^{1}$. Set $\bar{A}_{a^{1}}^{2}=\left\{i^{1}\right\}$, and set $\bar{A}_{a}^{2}=\emptyset$ for each $a \neq a^{1}$; these are the sets of students available to schools at the beginning of Step 2 . Each school $a \in A$ holds $\bar{C}^{a}\left(\bar{A}_{a}^{2}\right)$ and rejects all other students in $\bar{A}_{a}^{2}$.

Step $\ell$. Some student $i^{\ell} \in I$ not currently held by any school proposes to his most-preferred school that has not yet rejected him, $a^{\ell}$. Set $\bar{A}_{a^{\ell}}^{\ell+1}=\bar{A}_{a^{\ell}}^{\ell} \cup\left\{i^{\ell}\right\}$, and set $\bar{A}_{a}^{\ell+1}=\bar{A}_{a}^{\ell}$ for each $a \neq a^{\ell}$. Each school $a \in A$ holds $\bar{C}^{a}\left(\bar{A}_{a}^{\ell+1}\right)$ and rejects all other students in $\bar{A}_{a}^{\ell+1}$.

If at any Step $\ell+1$ no student is able to propose - that is, if all students not on hold have proposed to all schools they find acceptable - then the process terminates. The outcome of the cumulative offer process is the matching $\bar{\mu}$ which assigns each school $a \in A$ the students it holds at the end of the last step before termination: $\bar{\mu}_{a}=\bar{C}^{a}\left(\bar{A}_{a}^{\ell+1}\right)$. 
In our context, the cumulative offer process outcome is independent of the proposal order and is equal to the outcome of DA (see (Kominers and Sönmez 2012)).

We consider a modification of the cumulative offer process in which some students may be rejected as soon as they propose.

Definition. For sets of students $R_{a} \subseteq I$ ( $a \in A$ ), let the cumulative offer process with premature rejection under choice functions $\bar{C}$ be the following algorithm in which students propose to schools in a sequence of steps $\ell=1,2, \ldots$ :

Step 1. Some student $i^{1} \in I$ proposes to his favorite school $a^{1}$. Set $\bar{E}_{a^{1}}^{2}=\left\{i^{1}\right\}$, and set $\bar{E}_{a}^{2}=\emptyset$ for each $a \neq a^{1}$; these are the sets of students available to schools at the beginning of Step 2. Each school $a \in A$ holds $\left[\left(\bar{C}^{a}\left(\bar{E}_{a}^{2}\right)\right) \backslash R_{a}\right]$ and rejects all other students in $\bar{E}_{a}^{2}$.

Step $\ell$. Some student $i^{\ell} \in I$ not currently held by any school proposes to his most-preferred school that has not yet rejected him, $a^{\ell}$. Set $\bar{E}_{a^{\ell}}^{\ell+1}=\bar{E}_{a^{\ell}}^{\ell} \cup\left\{i^{\ell}\right\}$, and set $\bar{E}_{a}^{\ell+1}=\bar{E}_{a}^{\ell}$ for each $a \neq a^{\ell}$. Each school $a \in A$ holds $\left[\left(\bar{C}^{a}\left(\bar{E}_{a}^{\ell+1}\right)\right) \backslash R_{a}\right]$ and rejects all other students in $\bar{E}_{a}^{\ell+1}$.

If at any Step $\ell+1$ no student is able to propose - that is, if all students not on hold have proposed to all schools they find acceptable - then the process terminates. The outcome of the cumulative offer process with premature rejection is the matching $\bar{\mu}$ which assigns each school $a \in A$ the students it holds at the end of the last step before termination: $\bar{\mu}_{a}=\left[\left(\bar{C}^{a}\left(\bar{E}_{a}^{\ell+1}\right)\right) \backslash R_{a}\right]$.

Lemma 3. For each school $a \in A$, let $\bar{R}_{a}$ be the set of students rejected by a during the cumulative offer process under choice functions $\bar{C}$. For any choice of sets $R_{a} \subseteq \bar{R}_{a}(a \in A)$ and any sequence $\Sigma$ of student $\rightarrow$ school proposals $\Sigma=\left\langle\left(i^{1} \rightarrow a^{1}\right),\left(i^{2} \rightarrow a^{2}\right), \ldots\right\rangle$ that can arise in the cumulative offer process under choice functions $\bar{C}$ :

1. Proposal sequence $\Sigma$ is a valid proposal sequence in the cumulative offer process with premature rejection under choice functions $\bar{C}$.

2. Under proposal sequence $\Sigma$, the outcome of the cumulative offer process with premature rejection under choice functions $\bar{C}$, is the same as the outcome of the cumulative offer process under choice functions $\bar{C}$.

Proof. We denote by $A_{a}^{\ell}$ (resp. $E_{a}^{\ell}$ ) the sets of students available to each school $a \in A$ at the start of Step $\ell \geq 2$ of the cumulative offer process under choice functions $\bar{C}$ (resp. the cumulative offer process with premature rejection under choice functions $\bar{C}$ ).

It is clear that if proposal sequence $\Sigma$ is used for the first $\ell$ steps of each process, then $A_{a}^{m+1}=$ $E_{a}^{m+1}$ for all $m \leq \ell$. In this case, $\Sigma_{\ell+1}=\left(i^{\ell+1} \rightarrow a^{\ell+1}\right)$ is a valid proposal in Step $\ell+1$ of 
the cumulative offer process with premature rejection under choice functions $\bar{C} .{ }^{13}$ And if $\Sigma_{\ell+1}=$ $\left(i^{\ell+1} \rightarrow a^{\ell+1}\right)$ is proposed in Step $\ell+1$ of the cumulative offer process with premature rejection under choice functions $\bar{C}$, we have $E_{a}^{\ell+2}=E_{a}^{\ell+1} \cup\left\{i^{\ell+1}\right\}=A_{a}^{\ell+2}$ for each $a \in A$.

Now, $\Sigma_{1}$ is a valid proposal for the first step of each process. Inductive application of the preceding observations thus shows that $\Sigma$ is a valid proposal sequence in the cumulative offer process with premature rejection under choice functions $\bar{C}$. Moreover, under that proposal sequence we have $E_{a}^{\ell+1}=A_{a}^{\ell+1}$ for each $\ell$ and $a \in A$; hence, the eventual outcome of the cumulative offer process with premature rejection must be the same as the outcome of the cumulative offer process.

Now, we prove Propositions 1 and 2 using a completely parallel argument for the two results.

In the sequel, we assume the setup of either Section A.1 or Section A.2, let $C^{a}=\tilde{C}^{a}$ for all schools $a \neq a^{*}$, and let $\mu$ and $\tilde{\mu}$ respectively denote the cumulative offer process outcomes under the choice functions $C$ and $\tilde{C}$. We make use of an Adjustment Lemma, which is Lemma 1 for the case of Proposition 1 and Lemma 2 for the case of Proposition 2.

Proposition 6. Either

- $\mu_{a^{*}}=\tilde{\mu}_{a^{*}}$ - the same students are assigned to $a^{*}$ under $\mu$ and $\tilde{\mu}$-or

- $\mathfrak{n}_{a^{*}}(\tilde{\mu})>\mathfrak{n}_{a^{*}}(\mu)-a^{*}$ has strictly more walk-zone assignment under $\tilde{\mu}$ than under $\mu$.

Proof. We let $R_{a^{*}} \subseteq I$ be the set of students who are rejected from $a^{*}$ in both the cumulative offer process under the choice function $C$ and the cumulative offer process under the choice function $\tilde{C}$, and let $R_{a}=\emptyset$ for all $a \neq a^{*}$. By Lemma 3, $\mu$ and $\tilde{\mu}$ are the outcomes of the cumulative offer processes with premature rejection under the choice functions $C$ and $\tilde{C}$, respectively.

We now consider the cumulative offer processes with premature rejection under the choice functions $C$ and $\tilde{C}$, with orders of proposal chosen to be identical for the maximal number of steps possible ${ }^{14}$; we let $E_{a}^{\ell}$ and $\tilde{E}_{a}^{\ell}$ be the associated sets of effectively available students. If $\mu_{a} \neq \tilde{\mu}_{a}$, then there is some Step $\ell^{\prime}$ such that $\left[\left(C^{a^{*}}\left(E_{a^{*}}^{\ell^{\prime}+1}\right)\right) \backslash R_{a^{*}}\right] \neq\left[\left(\tilde{C}^{a^{*}}\left(\tilde{E}_{a^{*}}^{\ell^{\prime}+1}\right)\right) \backslash R_{a^{*}}\right]$; we let $\ell$ be the minimal such $\ell^{\prime}$. We let $n \equiv\left|I_{a^{*}} \cap\left[\left(C^{a^{*}}\left(E_{a^{*}}^{\ell+1}\right)\right) \backslash R_{a^{*}}\right]\right|$ be the number of walk-zone students held by $a^{*}$ at Step $\ell$ under choice function $C^{a^{*}}$, and let $\tilde{n} \equiv\left|I_{a^{*}} \cap\left[\left(\tilde{C}^{a^{*}}\left(\tilde{E}_{a^{*}}^{\ell+1}\right)\right) \backslash R_{a^{*}}\right]\right|$ be the number of walk-zone students held by $a^{*}$ at Step $\ell$ under choice function $\tilde{C}^{a^{*}}$.

By our choice of proposal orders and the minimality of $\ell$, we know that $E_{a^{*}}^{\ell+1}=\tilde{E}_{a^{*}}^{\ell+1}$; hence, $\tilde{C}^{a^{*}}\left(E_{a^{*}}^{\ell+1}\right)=\tilde{C}^{a^{*}}\left(\tilde{E}_{a^{*}}^{\ell+1}\right)$. As $\left[C^{a^{*}}\left(E_{a^{*}}^{\ell+1}\right) \backslash R_{a^{*}}\right] \neq\left[\tilde{C}^{a^{*}}\left(\tilde{E}_{a^{*}}^{\ell+1}\right) \backslash R_{a^{*}}\right]$, we know that

$$
C^{a^{*}}\left(E_{a^{*}}^{\ell+1}\right) \neq \tilde{C}^{a^{*}}\left(\tilde{E}_{a^{*}}^{\ell+1}\right)=\tilde{C}^{a^{*}}\left(E_{a^{*}}^{\ell+1}\right)
$$

\footnotetext{
${ }^{13}$ To see this, it suffices to note that $i^{\ell+1}$ is rejected in Step $\ell$ of the cumulative offer process under choice functions $\bar{C}$; hence,

$$
i^{\ell+1} \notin \bar{C}^{a}\left(A_{a}^{\ell+1}\right)
$$

for all $a \in A$. As $A_{a}^{\ell+1}=E_{a}^{\ell+1}$ for all all $a \in A$, (11) implies that $i^{\ell+1}$ is rejected in Step $\ell$ of the cumulative offer process with premature rejection under choice functions $\bar{C}$, and thus is free to propose in Step $\ell+1$.

${ }^{14}$ Such a maximum clearly exists, as the number of steps is bounded above by $|I| \cdot|A|$.
} 
We thus see from the first part of the Adjustment Lemma that there is a student $i \in I_{a^{*}}$ such that

$$
i \notin C^{a^{*}}\left(E_{a^{*}}^{\ell+1}\right) \quad \text { but } \quad i \in \tilde{C}^{a^{*}}\left(E_{a^{*}}^{\ell+1}\right)=\tilde{C}^{a^{*}}\left(\tilde{E}_{a^{*}}^{\ell+1}\right)
$$

it follows that

$$
\tilde{n}>n .
$$

We let $\tilde{i}_{\mathrm{m}} \in\left[I_{a^{*}} \cap\left[\left(\tilde{C}^{a^{*}}\left(\tilde{E}_{a^{*}}^{\ell+1}\right)\right) \backslash R_{a^{*}}\right]\right]$ be the student in the walk-zone of $a^{*}$ with the lowest ranking under $\pi^{o}$ of all students held in Step $\ell$ of the cumulative offer process with premature rejection under the choice functions $\tilde{C}$.

Claim. School $a^{*}$ never rejects student $\tilde{i}_{\mathrm{m}}$ during the running of the cumulative offer process with premature rejection under the choice functions $\tilde{C}$.

Proof. Since $\tilde{i}_{\mathrm{m}} \notin R_{a^{*}}$, it suffices to show that $\tilde{i}_{\mathrm{m}}$ is rejected by $a^{*}$ by Step $\ell$ of the cumulative offer process with premature rejection under the choice functions $C$. To see this, we suppose the contrary, that $\tilde{i}_{\mathrm{m}} \in\left[\left(C^{a^{*}}\left(E_{a^{*}}^{\ell+1}\right)\right) \backslash R_{a^{*}}\right] \subseteq C^{a^{*}}\left(E_{a^{*}}^{\ell+1}\right)$.

First, we observe that any

$$
i \in\left[I_{a^{*}} \cap\left[\left(\tilde{C}^{a^{*}}\left(\tilde{E}_{a^{*}}^{\ell+1}\right)\right) \backslash R_{a^{*}}\right]\right] \subseteq \tilde{E}_{a^{*}}^{\ell+1}=E_{a^{*}}^{\ell+1}
$$

ranked higher than $\tilde{i}_{\mathrm{m}}$ under $\pi^{o}$ must be in $C^{a^{*}}\left(E_{a^{*}}^{\ell+1}\right)$ : Otherwise, we would have $\tilde{i}_{\mathrm{m}}$ assigned to some slot $s \in S^{a^{*}}$ in the computation of $C^{a^{*}}\left(E_{a^{*}}^{\ell+1}\right)$, while

1. $i \notin C^{a^{*}}\left(E_{a^{*}}^{\ell+1}\right)$ (by assumption) and

2. $i \pi^{s} \tilde{i}_{\mathrm{m}}$ (because $i, \tilde{i}_{\mathrm{m}} \in I_{a^{*}}$ and $i \pi^{o} \tilde{i}_{\mathrm{m}}$ ).

We see from the definition of the function $C^{a^{*}}$ that this cannot happen $-\tilde{i}_{\mathrm{m}}$ cannot be assigned a slot before $i$.

The preceding observations imply that

$$
\left[I_{a^{*}} \cap\left[\left(\tilde{C}^{a^{*}}\left(\tilde{E}_{a^{*}}^{\ell+1}\right)\right) \backslash R_{a^{*}}\right]\right] \subseteq\left[\left(C^{a^{*}}\left(E_{a^{*}}^{\ell+1}\right)\right) \backslash R_{a^{*}}\right]
$$

Meanwhile, the second part of the Adjustment Lemma shows that $\left[\left(I \backslash I_{a^{*}}\right) \cap\left(\tilde{C}^{a^{*}}\left(E_{a^{*}}^{\ell+1}\right)\right)\right] \subseteq$ $\left[\left(I \backslash I_{a^{*}}\right) \cap\left(C^{a^{*}}\left(E_{a^{*}}^{\ell+1}\right)\right)\right]$; hence

$$
\left[\left(I \backslash I_{a^{*}}\right) \cap\left[\left(\tilde{C}^{a^{*}}\left(\tilde{E}_{a^{*}}^{\ell+1}\right)\right) \backslash R_{a^{*}}\right]\right]=\left[\left(I \backslash I_{a^{*}}\right) \cap\left[\left(\tilde{C}^{a^{*}}\left(E_{a^{*}}^{\ell+1}\right)\right) \backslash R_{a^{*}}\right]\right] \subseteq\left[\left(C^{a^{*}}\left(E_{a^{*}}^{\ell+1}\right)\right) \backslash R_{a^{*}}\right] .
$$

Combining (14) and (15), we see that $\left[\left(\tilde{C}^{a^{*}}\left(\tilde{E}_{a^{*}}^{\ell+1}\right)\right) \backslash R_{a^{*}}\right] \subseteq\left[\left(C^{a^{*}}\left(E_{a^{*}}^{\ell+1}\right)\right) \backslash R_{a^{*}}\right]$, so that we must have

$$
\left[\left(\tilde{C}^{a^{*}}\left(\tilde{E}_{a^{*}}^{\ell+1}\right)\right) \backslash R_{a^{*}}\right] \subsetneq\left[\left(C^{a^{*}}\left(E_{a^{*}}^{\ell+1}\right)\right) \backslash R_{a^{*}}\right]
$$

since $\left[\left(\tilde{C}^{a^{*}}\left(\tilde{E}_{a^{*}}^{\ell+1}\right)\right) \backslash R_{a^{*}}\right] \neq\left[\left(C^{a^{*}}\left(E_{a^{*}}^{\ell+1}\right)\right) \backslash R_{a^{*}}\right]$ by (12). But (16) is impossible from the definition of the function $\tilde{C}^{a^{*}}$, as it would imply that there is

1. some slot $s \in S^{a^{*}}$ not assigned a student in the computation of $\tilde{C}^{a^{*}}\left(\tilde{E}_{a^{*}}^{\ell+1}\right)$ and 
2. some student $i \in\left[E_{a^{*}}^{\ell+1} \backslash R_{a^{*}}\right]=\left[\tilde{E}_{a^{*}}^{\ell+1} \backslash R_{a^{*}}\right]$ not assigned to a slot of $a^{*}$ in the computation of $\tilde{C}^{a^{*}}\left(\tilde{E}_{a^{*}}^{\ell+1}\right)$

these two condtions cannot hold simultaneously because all slots rank all students as acceptable.

These observations show that the assumption that $\tilde{i}_{\mathrm{m}} \in\left[\left(C^{a^{*}}\left(E_{a^{*}}^{\ell+1}\right)\right) \backslash R_{a^{*}}\right]$ leads to a contradiction; hence, we have the claim.

The preceding claim implies that all walk-zone students $i \in\left[I_{a^{*}} \cap\left[\left(\tilde{C}^{a^{*}}\left(\tilde{E}_{a^{*}}^{\ell+1}\right)\right) \backslash R_{a^{*}}\right]\right]$ held by $a^{*}$ by Step $\ell$ of the cumulative offer process with premature rejection under the choice functions $\tilde{C}$ are held by $a^{*}$ in all remaining steps of the process, i.e.

$$
\tilde{\mu}_{a^{*}} \supseteq\left[I_{a^{*}} \cap\left[\left(\tilde{C}^{a^{*}}\left(\tilde{E}_{a^{*}}^{\ell+1}\right)\right) \backslash R_{a^{*}}\right]\right] .
$$

Thus, we see that

$$
\mathfrak{n}_{a^{*}}(\tilde{\mu}) \geq \tilde{n}
$$

Now, we let $i_{\mathrm{m}} \in\left[\left(I \backslash I_{a^{*}}\right) \cap\left[\left(C^{a^{*}}\left(E_{a^{*}}^{\ell+1}\right)\right) \backslash R_{a^{*}}\right]\right]$ be the student not in the walk-zone of $a^{*}$ with the lowest ranking under $\pi^{o}$ of all students held in Step $\ell$ of the cumulative offer process with premature rejection under the choice functions $C$.

Claim. School $a^{*}$ never rejects student $i_{\mathrm{m}}$ during the running of the cumulative offer process with premature rejection under the choice functions $C$.

Proof. The argument is completely analogous to that used to prove the preceding claim.

Since $i_{\mathrm{m}} \notin R_{a^{*}}$, it suffices to show that $i_{\mathrm{m}}$ is rejected by $a^{*}$ by Step $\ell$ of the cumulative offer process with premature rejection under the choice functions $\tilde{C}$. To see this, we suppose the contrary, that $i_{\mathrm{m}} \in\left[\left(\tilde{C}^{a^{*}}\left(\tilde{E}_{a^{*}}^{\ell+1}\right)\right) \backslash R_{a^{*}}\right] \subseteq \tilde{C}^{a^{*}}\left(\tilde{E}_{a^{*}}^{\ell+1}\right)$.

First, we observe that any

$$
i \in\left[\left(I \backslash I_{a^{*}}\right) \cap\left[\left(C^{a^{*}}\left(E_{a^{*}}^{\ell+1}\right)\right) \backslash R_{a^{*}}\right]\right] \subseteq E_{a^{*}}^{\ell+1}=\tilde{E}_{a^{*}}^{\ell+1}
$$

ranked higher than $i_{\mathrm{m}}$ under $\pi^{o}$ must be in $\tilde{C}^{a^{*}}\left(\tilde{E}_{a^{*}}^{\ell+1}\right)$ : Otherwise, we would have $i_{\mathrm{m}}$ assigned to some slot $s \in S^{a^{*}}$ in the computation of $\tilde{C}^{a^{*}}\left(\tilde{E}_{a^{*}}^{\ell+1}\right)$, while

1. $i \notin \tilde{C}^{a^{*}}\left(\tilde{E}_{a^{*}}^{\ell+1}\right)$ (by assumption) and

2. $i \pi^{s} i_{\mathrm{m}}$ (because $i, i_{\mathrm{m}} \in\left(I \backslash I_{a^{*}}\right)$ and $\left.i \pi^{o} i_{\mathrm{m}}\right)$.

We see from the definition of the function $\tilde{C}^{a^{*}}$ that this cannot happen- $i_{\mathrm{m}}$ cannot be assigned a slot before $i$.

The preceding observations imply that

$$
\left[\left(I \backslash I_{a^{*}}\right) \cap\left[\left(C^{a^{*}}\left(E_{a^{*}}^{\ell+1}\right)\right) \backslash R_{a^{*}}\right]\right] \subseteq\left[\left(\tilde{C}^{a^{*}}\left(\tilde{E}_{a^{*}}^{\ell+1}\right)\right) \backslash R_{a^{*}}\right] .
$$

Meanwhile, the first part of the Adjustment Lemma shows that $\left[I_{a^{*}} \cap\left(C^{a^{*}}\left(\tilde{E}_{a^{*}}^{\ell+1}\right)\right)\right] \subseteq\left[I_{a^{*}} \cap\right.$ $\left.\left(\tilde{C}^{a^{*}}\left(\tilde{E}_{a^{*}}^{\ell+1}\right)\right)\right]$; hence

$$
\left[I_{a^{*}} \cap\left[\left(C^{a^{*}}\left(E_{a^{*}}^{\ell+1}\right)\right) \backslash R_{a^{*}}\right]\right]=\left[I_{a^{*}} \cap\left[\left(C^{a^{*}}\left(\tilde{E}_{a^{*}}^{\ell+1}\right)\right) \backslash R_{a^{*}}\right]\right] \subseteq\left[\left(\tilde{C}^{a^{*}}\left(\tilde{E}_{a^{*}}^{\ell+1}\right)\right) \backslash R_{a^{*}}\right] .
$$


Combining (18) and (19), we see that $\left[\left(C^{a^{*}}\left(E_{a^{*}}^{\ell+1}\right)\right) \backslash R_{a^{*}}\right] \subseteq\left[\left(\tilde{C}^{a^{*}}\left(\tilde{E}_{a^{*}}^{\ell+1}\right)\right) \backslash R_{a^{*}}\right]$, so that we must have

$$
\left[\left(C^{a^{*}}\left(E_{a^{*}}^{\ell+1}\right)\right) \backslash R_{a^{*}}\right] \subsetneq\left[\left(\tilde{C}^{a^{*}}\left(\tilde{E}_{a^{*}}^{\ell+1}\right)\right) \backslash R_{a^{*}}\right]
$$

since $\left[\left(C^{a^{*}}\left(E_{a^{*}}^{\ell+1}\right)\right) \backslash R_{a^{*}}\right] \neq\left[\left(\tilde{C}^{a^{*}}\left(\tilde{E}_{a^{*}}^{\ell+1}\right)\right) \backslash R_{a^{*}}\right]$ by (12). But (16) is impossible from the definition of the function $C^{a^{*}}$, as it would imply that there is

1. some slot $s \in S^{a^{*}}$ not assigned a student in the computation of $C^{a^{*}}\left(E_{a^{*}}^{\ell+1}\right)$ and

2. some student $i \in\left[\tilde{E}_{a^{*}}^{\ell+1} \backslash R_{a^{*}}\right]=\left[E_{a^{*}}^{\ell+1} \backslash R_{a^{*}}\right]$ not assigned to a slot of $a^{*}$ in the computation of $C^{a^{*}}\left(E_{a^{*}}^{\ell+1}\right)$;

these two condtions cannot hold simultaneously because all slots rank all students as acceptable.

These observations show that the assumption that $i_{\mathrm{m}} \in\left[\left(\tilde{C}^{a^{*}}\left(\tilde{E}_{a^{*}}^{\ell+1}\right)\right) \backslash R_{a^{*}}\right]$ leads to a contradiction; hence, we have the claim.

The preceding claim implies that all non-walk-zone students $i \in\left[\left(I \backslash I_{a^{*}}\right) \cap\left[\left(C^{a^{*}}\left(E_{a^{*}}^{\ell+1}\right)\right) \backslash R_{a^{*}}\right]\right]$ held by $a^{*}$ by Step $\ell$ of the cumulative offer process with premature rejection under the choice functions $C$ are held by $a^{*}$ in all remaining steps of the process, i.e.

$$
\mu_{a^{*}} \supseteq\left[\left(I \backslash I_{a^{*}}\right) \cap\left[\left(C^{a^{*}}\left(E_{a^{*}}^{\ell+1}\right)\right) \backslash R_{a^{*}}\right]\right] .
$$

Thus, as the capacity of $a^{*}$ is fixed at $q_{a^{*}}$, we see that

$$
n \geq \mathfrak{n}_{a^{*}}(\mu) .
$$

Combining (13), (17), and (21), we see that

$$
\mathfrak{n}_{a^{*}}(\tilde{\mu}) \geq \tilde{n}>n \geq \mathfrak{n}_{a^{*}}(\mu)
$$

this proves the result.

\section{A.4 The Two-School Model}

\section{A.4.1 Preliminaries}

Matchings $\mu$ and $\tilde{\mu}$ are obtained as in Appendix A.3: Either one of the open slots is replaced with a walk-zone slot, or the precedence position of a walk-zone slot is switched with that of a subsequent open slot to obtain $\tilde{C}$ from $C$, and $\tilde{\mu}$ and $\mu$ are, respectively, the associated cumulative offer process outcomes.

Lemma 4. We have $\left|\tilde{\mu}_{a}\right|=\left|\mu_{a}\right|$ and $\left|\tilde{\mu}_{b}\right|=\left|\mu_{b}\right|$. That is, the number of slots filled at each school is the same under $\mu$ as under $\tilde{\mu}$.

Proof. If both of the schools $a$ and $b$ have an empty slot under either matching, stability implies that all students get their first choices under each matching; hence $\tilde{\mu}=\mu$ and the result holds immediately. Likewise, if neither school has an empty slot under either matching, the result holds 
immediately since then $\left|\tilde{\mu}_{a}\right|=\left|\mu_{a}\right|=\left|S^{a}\right|$ and $\left|\tilde{\mu}_{b}\right|=\left|\mu_{b}\right|=\left|S^{b}\right|$. Hence the only non-trivial case is when, under one of the matchings, one school is full but the other is not.

Without loss of generality, we suppose that under matching $\mu$, school $a$ has an empty slot whereas school $b$ has all its slots full. Then not only does each student who is assigned a slot at school $b$ under matching $\mu$ prefer school $b$ to school $a$, but also there are at least as many students with a first choice of school $b$ as the number of slots at school $b$. Thus by stability school $b$ must fill all its slots under matching $\tilde{\mu}$ as well; hence, $\left|\tilde{\mu}_{b}\right|=\left|\mu_{b}\right|=\left|S^{b}\right|$. By assumption,

- there are at least as many slots as students, and

- all students find both schools acceptable;

therefore, we see that

$$
\left|\tilde{\mu}_{a}\right|=|I|-\left|\tilde{\mu}_{b}\right|=|I|-\left|\mu_{b}\right|=\left|\mu_{a}\right| .
$$

This observation completes the proof.

\section{A.4.2 Proof of Propositions 3 and 4}

We prove Propositions 3 and 4 using a completely parallel argument for the two results. We make use of an Adjustment Proposition, which is is Proposition 1 for the case of Proposition 3, and Proposition 2 for the case of Proposition 4.

Proposition 7. There is weakly more walk-zone assignment under $\tilde{\mu}$ than under $\mu$, that is,

$$
\mathfrak{n}_{a}(\tilde{\mu})+\mathfrak{n}_{b}(\tilde{\mu}) \geq \mathfrak{n}_{a}(\mu)+\mathfrak{n}_{b}(\mu) .
$$

Proof. Without loss of generality, we assume the priority structure of school $a$ has changed (i.e. that $a=a^{*}$ in the setup of Appendix A.3).

If $\tilde{\mu}_{a}=\mu_{a}$, then we have

$$
\tilde{\mu}_{b}=I \backslash \tilde{\mu}_{a}=I \backslash \mu_{a}=\mu_{b},
$$

as by assumption

- there are at least as many slots as students, and

- all students find both schools acceptable.

Thus, in this case the result is immediate.

If $\tilde{\mu}_{a} \neq \mu_{a}$

$$
\left|\tilde{\mu}_{a} \cap I_{a}\right|=\mathfrak{n}_{a}(\tilde{\mu})>\mathfrak{n}_{a}(\mu)=\left|\mu_{a} \cap I_{a}\right|
$$

by the Adjustment Proposition. Therefore Lemma 4 implies that

$$
\left|\tilde{\mu}_{a} \cap\left(I \backslash I_{a}\right)\right|=\left|\tilde{\mu}_{a}\right|-\left|\tilde{\mu}_{a} \cap I_{a}\right|<\left|\mu_{a}\right|-\left|\mu_{a} \cap I_{a}\right|=\left|\mu_{a} \cap\left(I \backslash I_{a}\right)\right|,
$$

which in turn implies that

$$
\left|\tilde{\mu}_{a} \cap I_{b}\right|<\left|\mu_{a} \cap I_{b}\right|
$$


as $I \backslash I_{a}=I_{b}$ by assumption. Thus, we see that

$$
\mathfrak{n}_{b}(\tilde{\mu})=\left|\tilde{\mu}_{b} \cap I_{b}\right|=\left|I_{b}\right|-\left|\tilde{\mu}_{a} \cap I_{b}\right|>\left|I_{b}\right|-\left|\mu_{a} \cap I_{b}\right|=\left|\mu_{b} \cap I_{b}\right|=\mathfrak{n}_{b}(\mu)
$$

as all students (and in particular all students in $I_{b}$ ) are matched under both $\mu$ and $\tilde{\mu}$. Hence in this case

$$
\mathfrak{n}_{a}(\tilde{\mu})+\mathfrak{n}_{b}(\tilde{\mu})>\mathfrak{n}_{a}(\mu)+\mathfrak{n}_{b}(\mu)
$$

this completes the proof.

\section{A.4.3 Proof of Proposition 5}

Let $r_{a}^{1}$ denote the number of students who rank school $a$ as first choice, and let $r_{b}^{1}$ denote the number of students who rank school $b$ as first choice.

We can obtain the outcome of the DA by either the student proposing deferred acceptance algorithm or the cumulative offer process. We utilize the former in this proof.

By assumption, $\left|S^{a}\right|+\left|S^{b}\right| \geq|I|$. Thus, as each student has a first choice,

$$
\left|S^{a}\right|+\left|S^{b}\right| \geq r_{a}^{1}+r_{b}^{1}
$$

Hence, either:

1. $\left|S^{a}\right| \geq r_{a}^{1}$ and $\left|S^{b}\right| \geq r_{b}^{1}$, or

2. $\left|S^{a}\right|>r_{a}^{1}$ and $\left|S^{b}\right|<r_{b}^{1}$, or

3. $\left|S^{a}\right|<r_{a}^{1}$ and $\left|S^{b}\right|>r_{b}^{1}$.

In the first case, the student proposing deferred acceptance algorithm terminates in one step and all students receive their first choices under both $\mu$ and $\tilde{\mu}$. Thus, the result is immediate in this case.

The analyses of the second and third cases are analogous, so it suffices to consider the case that $\left|S^{a}\right|>r_{a}^{1}$ and $\left|S^{b}\right|<r_{b}^{1}$.

Claim. For this case, under both $\mu$ and $\tilde{\mu}$,

- the number of students receiving their first choices is equal to $\left|S^{b}\right|+r_{a}^{1}$, and

- the number of students receiving their second choices is equal to $r_{b}^{1}-\left|S^{b}\right|$.

Proof. We consider the construction of either $\mu$ or $\tilde{\mu}$ through the student proposing deferred acceptance algorithm, and observe that school $b$ receives $r_{b}^{1}>\left|S^{b}\right|$ offers in Step 1, holding $\left|S^{b}\right|$ of these while rejecting $r_{b}^{1}-\left|S^{b}\right|$. School $a$, meanwhile, receives $r_{a}^{1}<\left|S^{a}\right|$ offers and holds all of them. In Step 2, all students rejected by school $b$ apply to school $a$, bringing the total number of applicants 
at school $a$ to $r_{a}^{1}+\left(r_{b}^{1}-\left|S^{b}\right|\right)$. As $r_{a}^{1}+\left(r_{b}^{1}-\left|S^{b}\right|\right) \leq\left|S^{a}\right|$ by assumption, no student is rejected by school $a$, and the algorithm terminates in Step 2. ${ }^{15}$ Hence, under both $\mu$ and $\tilde{\mu}$,

- $\left|S^{b}\right|$ students are assigned to school $b$ as their first choice,

- $r_{a}^{1}$ students are assigned to school $a$ as their first choice, and

- $r_{b}^{1}-\left|S^{b}\right|$ students are assigned to school $a$ as their second choice.

These observations show the claim.

The preceding claim shows the result for the second case; since an analogous argument shows the result for the third case, this completes the proof.

\footnotetext{
${ }^{15}$ While it is well-known that stability may be in conflict with Pareto efficiency in general school choice environments (cf. Balinski and Sönmez (1999), Ergin (2002), Abdulkadiroğlu and Sönmez (2003), Kesten (2006), and Kesten (2010)), the above argument shows that DA is Pareto efficient in our two-school environment.
} 
Table 1. Difference between the Current Boston Mechanism and Alternative Walk Zone Splits

\begin{tabular}{|c|c|c|c|c|c|c|c|c|c|}
\hline & \multicolumn{3}{|c|}{$\begin{array}{l}\text { Grade K1 } \\
\text { Difference relative to current BPS }\end{array}$} & \multicolumn{3}{|c|}{$\begin{array}{l}\text { Grade K2 } \\
\text { Difference relative to current BPS }\end{array}$} & \multicolumn{3}{|c|}{$\begin{array}{c}\text { Grade } 6 \\
\text { Difference relative to current BPS }\end{array}$} \\
\hline & $\begin{array}{l}\text { \# students } \\
(1)\end{array}$ & $\begin{array}{c}0 \% \text { Walk } \\
(2)\end{array}$ & $\begin{array}{l}\text { 100\% Walk } \\
\text { (3) }\end{array}$ & $\begin{array}{l}\text { \# students } \\
\text { (4) }\end{array}$ & $\begin{array}{c}\text { 0\% Walk } \\
\text { (5) }\end{array}$ & $\begin{array}{l}100 \% \text { Walk } \\
(6)\end{array}$ & $\begin{array}{l}\text { \# students } \\
(7)\end{array}$ & $\begin{array}{c}0 \% \text { Walk } \\
(8)\end{array}$ & $\begin{array}{l}\text { 100\% Walk } \\
\text { (9) }\end{array}$ \\
\hline 2009 & 1770 & $\begin{array}{l}46 \\
3 \%\end{array}$ & $\begin{array}{l}336 \\
19 \%\end{array}$ & 1715 & $\begin{array}{l}28 \\
2 \%\end{array}$ & $\begin{array}{l}343 \\
20 \%\end{array}$ & 2348 & $\begin{array}{l}54 \\
2 \%\end{array}$ & $\begin{array}{l}205 \\
9 \%\end{array}$ \\
\hline 2010 & 1977 & $\begin{array}{l}68 \\
3 \%\end{array}$ & $\begin{array}{l}392 \\
20 \%\end{array}$ & 1902 & $\begin{array}{l}62 \\
3 \%\end{array}$ & $\begin{array}{l}269 \\
14 \%\end{array}$ & 2308 & $\begin{array}{l}41 \\
2 \%\end{array}$ & $\begin{array}{l}171 \\
7 \%\end{array}$ \\
\hline 2011 & 2071 & $\begin{array}{l}50 \\
2 \%\end{array}$ & $\begin{array}{l}387 \\
19 \%\end{array}$ & 1821 & $\begin{array}{l}90 \\
5 \%\end{array}$ & $\begin{array}{l}293 \\
16 \%\end{array}$ & 2073 & $\begin{array}{c}4 \\
0 \%\end{array}$ & $\begin{array}{l}225 \\
11 \%\end{array}$ \\
\hline 2012 & 2515 & $\begin{array}{l}88 \\
3 \%\end{array}$ & $\begin{array}{l}504 \\
20 \%\end{array}$ & 2301 & $\begin{array}{c}101 \\
4 \%\end{array}$ & $\begin{array}{l}403 \\
18 \%\end{array}$ & 2057 & $\begin{array}{l}24 \\
1 \%\end{array}$ & $\begin{array}{l}247 \\
12 \%\end{array}$ \\
\hline All & 8333 & $\begin{array}{c}252 \\
3 \% \\
\end{array}$ & $\begin{array}{c}1619 \\
19 \%\end{array}$ & 7739 & $\begin{array}{c}281 \\
4 \% \\
\end{array}$ & $\begin{array}{c}1308 \\
17 \%\end{array}$ & 8786 & $\begin{array}{c}123 \\
1 \%\end{array}$ & $\begin{array}{l}848 \\
10 \% \\
\end{array}$ \\
\hline
\end{tabular}

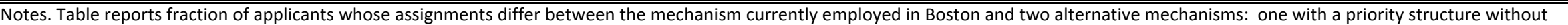

walk-zone priorities at any seats ( $0 \%$ Walk), and the other with a priority structure with walk-zone priorities at all seats (100\% Walk). 
Table 2. Number of Students Assigned to School in Walk Zone, One Lottery Number

\begin{tabular}{|c|c|c|c|c|c|c|c|}
\hline & \multirow{2}{*}{$\begin{array}{c}\text { Priorities = } \\
\text { \%\% Walk } \\
\text { (1) }\end{array}$} & \multicolumn{5}{|c|}{$\frac{\text { Priorities }=50 \% \text { Walk }}{\text { Changing Precedence }}$} & \multirow{2}{*}{$\begin{array}{c}\text { Priorities }= \\
100 \% \text { Walk } \\
\text { (7) }\end{array}$} \\
\hline & & $\begin{array}{l}\text { Walk-Open } \\
(2)\end{array}$ & $\begin{array}{c}\text { Actual BPS } \\
\text { (3) }\end{array}$ & $\begin{array}{c}\text { Rotating } \\
\text { (4) }\end{array}$ & $\begin{array}{c}\text { Compromise } \\
\text { (5) } \\
\end{array}$ & $\begin{array}{c}\text { Open-Walk } \\
(6) \\
\end{array}$ & \\
\hline & \multicolumn{7}{|c|}{ I. Grade K1 } \\
\hline \multirow[t]{2}{*}{ Walk Zone } & 3849 & 3879 & 3930 & 4080 & 4227 & 4570 & 4787 \\
\hline & $46.2 \%$ & $46.6 \%$ & $47.2 \%$ & $49.0 \%$ & $50.7 \%$ & $54.8 \%$ & $57.4 \%$ \\
\hline \multirow[t]{2}{*}{ Outside Walk Zone } & 2430 & 2399 & 2353 & 2187 & 2044 & 1695 & 1468 \\
\hline & $29.2 \%$ & $28.8 \%$ & $28.2 \%$ & $26.2 \%$ & $24.5 \%$ & $20.3 \%$ & $17.6 \%$ \\
\hline \multirow[t]{3}{*}{ Unassigned } & 2054 & 2055 & 2050 & 2066 & 2062 & 2068 & 2078 \\
\hline & $24.6 \%$ & $24.7 \%$ & $24.6 \%$ & $24.8 \%$ & $24.7 \%$ & $24.8 \%$ & $24.9 \%$ \\
\hline & \multicolumn{7}{|c|}{ II. Grade K2 } \\
\hline \multirow[t]{2}{*}{ Walk Zone } & 3651 & 3685 & 3753 & 3842 & 3900 & 4214 & 4374 \\
\hline & $47.2 \%$ & $47.6 \%$ & $48.5 \%$ & $49.6 \%$ & $50.4 \%$ & $54.5 \%$ & $56.5 \%$ \\
\hline \multirow[t]{2}{*}{ Outside Walk Zone } & 2799 & 2764 & 2694 & 2601 & 2538 & 2214 & 2036 \\
\hline & $36.2 \%$ & $35.7 \%$ & $34.8 \%$ & $33.6 \%$ & $32.8 \%$ & $28.6 \%$ & $26.3 \%$ \\
\hline \multirow{3}{*}{ Unassigned } & 1289 & 1290 & 1292 & 1296 & 1301 & 1311 & 1329 \\
\hline & $16.7 \%$ & $16.7 \%$ & $16.7 \%$ & $16.7 \%$ & $16.8 \%$ & $16.9 \%$ & $17.2 \%$ \\
\hline & \multicolumn{7}{|c|}{ III. Grade 6} \\
\hline \multirow[t]{2}{*}{ Walk Zone } & 3439 & 3476 & 3484 & 3542 & 3657 & 3797 & 3907 \\
\hline & $39.1 \%$ & $39.6 \%$ & $39.7 \%$ & $40.3 \%$ & $41.6 \%$ & $43.2 \%$ & $44.5 \%$ \\
\hline \multirow[t]{2}{*}{ Outside Walk Zone } & 4782 & 4750 & 4743 & 4686 & 4561 & 4419 & 4309 \\
\hline & $54.4 \%$ & $54.1 \%$ & $54.0 \%$ & $53.3 \%$ & $51.9 \%$ & $50.3 \%$ & $49.0 \%$ \\
\hline \multirow[t]{2}{*}{ Unassigned } & 565 & 560 & 559 & 558 & 568 & 570 & 570 \\
\hline & $6.4 \%$ & $6.4 \%$ & $6.4 \%$ & $6.4 \%$ & $6.5 \%$ & $6.5 \%$ & $6.5 \%$ \\
\hline
\end{tabular}

Notes. Table reports fraction of applicants assigned to walk-zone schools under several alternative assignment procedures from 2009-2012. 0\% Walk implements the studentproposing deferred acceptance mechanism with no walk zone priority; $100 \%$ implements the student-proposing deferred acceptance mechanism with all slots having walkzone priority. Columns (2)-(6) hold the 50-50 school seat split fixed. Walk-Open implements the precedence order in which all walk-zone slots are ahead of open slots.

Actual BPS implements the current BPS system. Rotating implements the precedence ordering alternating between walk-zone and open slots. Compromise implements the precedence order in which exactly half of the walk-zone slots come before all open slots, which are in turn followed by the second half of the walk-zone slots (25W-500$25 \mathrm{~W})$. Open-Walk implements the precedence order in which all open slots are ahead of walk-zone slots. 
Table 3. Number of Students Assigned to School in Walk Zone, Two Lottery Numbers

\begin{tabular}{|c|c|c|c|c|c|}
\hline & \multirow{2}{*}{$\begin{array}{c}\text { Priorities }= \\
\underline{0 \% \text { Walk }} \\
\text { (1) }\end{array}$} & \multicolumn{3}{|c|}{$\begin{array}{l}\text { Priorities }=50 \% \text { Walk } \\
\text { Changing Precedence }\end{array}$} & \multirow{2}{*}{$\begin{array}{c}\text { Priorities }= \\
\underline{100 \% \text { Walk }} \\
\text { (5) }\end{array}$} \\
\hline & & $\begin{array}{c}\text { Walk-Open: } \\
\text { Two Lotteries } \\
\text { (2) }\end{array}$ & $\begin{array}{l}\text { Rotating: Two } \\
\text { Lotteries } \\
(3) \\
\end{array}$ & $\begin{array}{c}\text { Open-Walk: } \\
\text { Two Lotteries } \\
\text { (4) }\end{array}$ & \\
\hline & \multicolumn{5}{|c|}{ 1. Grade K1 } \\
\hline \multirow[t]{2}{*}{ Walk Zone } & 3849 & 4034 & 4306 & 4556 & 4787 \\
\hline & $46.2 \%$ & $48.4 \%$ & $51.7 \%$ & $54.7 \%$ & $57.4 \%$ \\
\hline \multirow[t]{2}{*}{ Outside Walk Zone } & 2430 & 2217 & 1944 & 1709 & 1468 \\
\hline & $29.2 \%$ & $26.6 \%$ & $23.3 \%$ & $20.5 \%$ & $17.6 \%$ \\
\hline \multirow[t]{3}{*}{ Unassigned } & 2054 & 2082 & 2083 & 2068 & 2078 \\
\hline & $24.6 \%$ & $25.0 \%$ & $25.0 \%$ & $24.8 \%$ & $24.9 \%$ \\
\hline & \multicolumn{5}{|c|}{ II. Grade K2 } \\
\hline \multirow[t]{2}{*}{ Walk Zone } & 3651 & 3880 & 4064 & 4210 & 4374 \\
\hline & $47.2 \%$ & $50.1 \%$ & $52.5 \%$ & $54.4 \%$ & $56.5 \%$ \\
\hline \multirow[t]{2}{*}{ Outside Walk Zone } & 2799 & 2539 & 2352 & 2220 & 2036 \\
\hline & $36.2 \%$ & $32.8 \%$ & $30.4 \%$ & $28.7 \%$ & $26.3 \%$ \\
\hline \multirow[t]{3}{*}{ Unassigned } & 1289 & 1320 & 1323 & 1309 & 1329 \\
\hline & $16.7 \%$ & $17.1 \%$ & $17.1 \%$ & $16.9 \%$ & $17.2 \%$ \\
\hline & \multicolumn{5}{|c|}{ III. Grade 6} \\
\hline \multirow[t]{2}{*}{ Walk Zone } & 3439 & 3516 & 3726 & 3784 & 3907 \\
\hline & $39.1 \%$ & $40.0 \%$ & $42.4 \%$ & $43.1 \%$ & $44.5 \%$ \\
\hline \multirow[t]{2}{*}{ Outside Walk Zone } & 4782 & 4655 & 4461 & 4415 & 4309 \\
\hline & $54.4 \%$ & $53.0 \%$ & $50.8 \%$ & $50.3 \%$ & $49.0 \%$ \\
\hline \multirow[t]{2}{*}{ Unassigned } & 565 & 615 & 599 & 587 & 570 \\
\hline & $6.4 \%$ & $7.0 \%$ & $6.8 \%$ & $6.7 \%$ & $6.5 \%$ \\
\hline \multicolumn{6}{|c|}{$\begin{array}{l}\text { Notes. Table reports fraction of applicants assigned to walk-zone schools under several alternative assignment procedures from } \\
2009-2012.0 \% \text { Walk implements the student-proposing deferred acceptance mechanism with no walk zone priority; } 100 \% \\
\text { implements the student-proposing deferred acceptance mechanism with all slots having walk-zone priority. Columns (2)-(4) hold } \\
\text { the } 50-50 \text { school seat split fixed. Walk-Open implements the precedence order in which all walk-zone slots are ahead of open } \\
\text { slots, but uses two different lottery numbers for walk and open seats. Rotating implements the precedence ordering alternating } \\
\text { between open and walk-zone slots, in that order. Open-Walk implements the precedence order in which all open slots are ahead } \\
\text { of walk-zone slots, but uses two different lottery numbers for walk and open. The same lottery numbers are used for each } \\
\text { simulation. }\end{array}$} \\
\hline
\end{tabular}


Table 4. What Policy Was Being Implemented in Boston?

\begin{tabular}{|c|c|c|c|c|c|c|c|}
\hline & $\begin{array}{c}\text { Priorities }=0 \% \\
\text { Walk }\end{array}$ & $\begin{array}{c}\text { Priorities }=5 \% \\
\text { Walk } \\
\text { Open-Walk: } \\
\text { One Lottery } \\
\text { (2) }\end{array}$ & Actual BPS & $\begin{array}{c}\text { Priorities }= \\
\underline{10 \% \text { Walk }} \\
\text { Rotating: Two } \\
\text { Lotteries } \\
\text { (4) }\end{array}$ & $\begin{array}{c}\frac{\text { Priorities }=}{10 \% \text { Walk }} \\
\underline{\text { Open-Walk: }} \\
\text { One Lottery } \\
\text { (5) }\end{array}$ & $\begin{array}{c}\text { Priorities }= \\
\underline{25 \% \text { Walk }} \\
\text { Rotating: Two } \\
\text { Lotteries } \\
(6)\end{array}$ & $\begin{array}{c}\text { Priorities = } \\
25 \% \text { Walk } \\
\text { Open-Walk: } \\
\text { One Lottery } \\
\text { (7) }\end{array}$ \\
\hline & & & & I. Grade K1 & & & \\
\hline \multirow[t]{2}{*}{ Walk Zone } & 3849 & 3906 & 3930 & 3892 & 3965 & 4021 & 4088 \\
\hline & $46.2 \%$ & $46.9 \%$ & $47.2 \%$ & $46.7 \%$ & $47.6 \%$ & $48.3 \%$ & $49.1 \%$ \\
\hline \multirow[t]{2}{*}{ Outside Walk Zone } & 2430 & 2369 & 2353 & 2384 & 2304 & 2250 & 2183 \\
\hline & $29.2 \%$ & $28.4 \%$ & $28.2 \%$ & $28.6 \%$ & $27.6 \%$ & $27.0 \%$ & $26.2 \%$ \\
\hline \multirow[t]{3}{*}{ Unassigned } & 2054 & 2058 & 2050 & 2057 & 2064 & 2062 & 2062 \\
\hline & $24.6 \%$ & $24.7 \%$ & $24.6 \%$ & $24.7 \%$ & $24.8 \%$ & $24.7 \%$ & $24.7 \%$ \\
\hline & & & & II. Grade K2 & & & \\
\hline \multirow[t]{2}{*}{ Walk Zone } & 3651 & 3692 & 3753 & 3671 & 3743 & 3746 & 3825 \\
\hline & $47.2 \%$ & $47.7 \%$ & $48.5 \%$ & $47.4 \%$ & $48.4 \%$ & $48.4 \%$ & $49.4 \%$ \\
\hline \multirow[t]{2}{*}{ Outside Walk Zone } & 2799 & 2757 & 2694 & 2772 & 2702 & 2693 & 2606 \\
\hline & $36.2 \%$ & $35.6 \%$ & $34.8 \%$ & $35.8 \%$ & $34.9 \%$ & $34.8 \%$ & $33.7 \%$ \\
\hline \multirow[t]{3}{*}{ Unassigned } & 1289 & 1290 & 1292 & 1296 & 1294 & 1300 & 1308 \\
\hline & $16.7 \%$ & $16.7 \%$ & $16.7 \%$ & $16.7 \%$ & $16.7 \%$ & $16.8 \%$ & $16.9 \%$ \\
\hline & & & & III. Grade 6 & & & \\
\hline \multirow[t]{2}{*}{ Walk Zone } & 3439 & 3461 & 3484 & 3466 & 3496 & 3511 & 3546 \\
\hline & $39.1 \%$ & $39.4 \%$ & $39.7 \%$ & $39.4 \%$ & $39.8 \%$ & $40.0 \%$ & $40.4 \%$ \\
\hline \multirow[t]{2}{*}{ Outside Walk Zone } & 4782 & 4751 & 4743 & 4745 & 4715 & 4694 & 4655 \\
\hline & $54.4 \%$ & $54.1 \%$ & $54.0 \%$ & $54.0 \%$ & $53.7 \%$ & $53.4 \%$ & $53.0 \%$ \\
\hline \multirow[t]{2}{*}{ Unassigned } & 565 & 574 & 559 & 575 & 575 & 581 & 585 \\
\hline & $6.4 \%$ & $6.5 \%$ & $6.4 \%$ & $6.5 \%$ & $6.5 \%$ & $6.6 \%$ & $6.7 \%$ \\
\hline
\end{tabular}

Notes. Table reports fraction of applicants assigned to walk-zone schools under several alternative assignment procedures from 2009-2012.0\% Walk

implements the student-proposing deferred acceptance mechanism with no walk zone priority. Rotating implements the precedence ordering alternating between open and walk-zone, but uses two different lottery numbers for walk and open seats. Open-Walk implements the precedence order in which all open slots are ahead of walk-zone slots. For $10 \%$ Walk priority, Rotating is implemented as 9 open seats, followed by 1 walk seat for each block of 10 school seats. If the number of school seats is not an exact multiple of 10 , let $X$ be the number of seats modulo 10 . If $X$ is greater than 5 , then the last block is

implemented as (X-1) open seats, followed by 1 walk seat. For $25 \%$ Walk priority, Rotating is implemented as 3 open seats, followed by 1 walk seat for each block of 4 school seats. The same rule as used for 10 seats is used for the last block of 4 seats if schools seats are not an exact multiple of 4 . The same lottery numbers are used for each simulation. 
Table 5. Rank of Assignment Received by Students

\begin{tabular}{|c|c|c|c|c|c|}
\hline \multirow{4}{*}{$\begin{array}{l}\text { Choice } \\
\text { Received }\end{array}$} & \multirow{3}{*}{$\frac{\text { Priorities }=}{\underline{0 \% \text { Walk }}}$} & \multirow{2}{*}{\multicolumn{3}{|c|}{$\begin{array}{l}\text { Priorities }=50 \% \text { Walk } \\
\text { Changing Precedence }\end{array}$}} & \multirow{3}{*}{$\begin{array}{l}\text { Priorities }= \\
100 \% \text { Walk }\end{array}$} \\
\hline & & & & & \\
\hline & & \multicolumn{3}{|c|}{ Rotating: Two } & \\
\hline & (1) & $\begin{array}{c}\text { Actual BPS } \\
\text { (2) }\end{array}$ & $\begin{array}{c}\text { Lotteries } \\
\text { (3) }\end{array}$ & $\begin{array}{c}\text { Open-Walk } \\
\text { (4) }\end{array}$ & $(5)$ \\
\hline & \multicolumn{5}{|c|}{ I. Grade K1 } \\
\hline 1 & $44 \%$ & $44 \%$ & $43 \%$ & $44 \%$ & $43 \%$ \\
\hline 2 & $13 \%$ & $13 \%$ & $13 \%$ & $14 \%$ & $13 \%$ \\
\hline 3 & $8 \%$ & $8 \%$ & $8 \%$ & $8 \%$ & $9 \%$ \\
\hline 4 & $5 \%$ & $5 \%$ & $4 \%$ & $4 \%$ & $5 \%$ \\
\hline 5 & $2 \%$ & $2 \%$ & $2 \%$ & $2 \%$ & $2 \%$ \\
\hline 6 & $1 \%$ & $1 \%$ & $1 \%$ & $1 \%$ & $1 \%$ \\
\hline 7 & $1 \%$ & $1 \%$ & $1 \%$ & $1 \%$ & $1 \%$ \\
\hline 8 & $0 \%$ & $1 \%$ & $0 \%$ & $1 \%$ & $1 \%$ \\
\hline 9 & $0 \%$ & $0 \%$ & $0 \%$ & $0 \%$ & $0 \%$ \\
\hline 10 & $0 \%$ & $0 \%$ & $0 \%$ & $0 \%$ & $0 \%$ \\
\hline \multirow{2}{*}{$\begin{array}{l}\text { Unassigned or } \\
\text { Admin. Assigned }\end{array}$} & $25 \%$ & $25 \%$ & $25 \%$ & $25 \%$ & $25 \%$ \\
\hline & \multicolumn{5}{|c|}{ II. Grade K2 } \\
\hline 1 & $49 \%$ & $49 \%$ & $49 \%$ & $49 \%$ & $49 \%$ \\
\hline 2 & $16 \%$ & $16 \%$ & $16 \%$ & $16 \%$ & $16 \%$ \\
\hline 3 & $10 \%$ & $10 \%$ & $10 \%$ & $10 \%$ & $10 \%$ \\
\hline 4 & $4 \%$ & $4 \%$ & $4 \%$ & $4 \%$ & $4 \%$ \\
\hline 5 & $2 \%$ & $2 \%$ & $2 \%$ & $2 \%$ & $2 \%$ \\
\hline 6 & $1 \%$ & $1 \%$ & $1 \%$ & $1 \%$ & $1 \%$ \\
\hline 7 & $0 \%$ & $0 \%$ & $1 \%$ & $0 \%$ & $0 \%$ \\
\hline 8 & $0 \%$ & $0 \%$ & $0 \%$ & $0 \%$ & $0 \%$ \\
\hline 9 & $0 \%$ & $0 \%$ & $0 \%$ & $0 \%$ & $0 \%$ \\
\hline 10 & $0 \%$ & $0 \%$ & $0 \%$ & $0 \%$ & $0 \%$ \\
\hline \multirow{3}{*}{$\begin{array}{l}\text { Unassigned or } \\
\text { Admin. Assigned }\end{array}$} & $17 \%$ & $17 \%$ & $17 \%$ & $17 \%$ & $17 \%$ \\
\hline & & & & & \\
\hline & \multicolumn{5}{|c|}{ III. Grade 6} \\
\hline 1 & $66 \%$ & $66 \%$ & $65 \%$ & $65 \%$ & $65 \%$ \\
\hline 2 & $18 \%$ & $18 \%$ & $18 \%$ & $18 \%$ & $18 \%$ \\
\hline 3 & $7 \%$ & $7 \%$ & $8 \%$ & $7 \%$ & $7 \%$ \\
\hline 4 & $2 \%$ & $2 \%$ & $2 \%$ & $2 \%$ & $2 \%$ \\
\hline 5 & $1 \%$ & $1 \%$ & $1 \%$ & $1 \%$ & $1 \%$ \\
\hline 6 & $0 \%$ & $0 \%$ & $0 \%$ & $0 \%$ & $0 \%$ \\
\hline 7 & $0 \%$ & $0 \%$ & $0 \%$ & $0 \%$ & $0 \%$ \\
\hline 8 & $0 \%$ & $0 \%$ & $0 \%$ & $0 \%$ & $0 \%$ \\
\hline 9 & $0 \%$ & $0 \%$ & $0 \%$ & $0 \%$ & $0 \%$ \\
\hline 10 & $0 \%$ & $0 \%$ & $0 \%$ & $0 \%$ & $0 \%$ \\
\hline Unassigned or & $6 \%$ & $6 \%$ & $7 \%$ & $6 \%$ & $6 \%$ \\
\hline Admin. Assigned & & & & & \\
\hline
\end{tabular}

Notes. Table reports the distribution of choice ranks arising under different priority and precedence policies. Unassigned or Adminstrative Assignment means student is not assigned to any of her listed choices; some students will be adminstratively assigned after Round 1. 


\section{References}

AbdulkadiroĞlu, A., P. A. Pathak, and A. E. Roth (2009): "Strategy-proofness versus Efficiency in Matching with Indifferences: Redesigning the New York City High School Match," American Economic Review, 99(5), 1954-1978.

AbdulkadiroĞlu, A., P. A. Pathak, A. E. Roth, and T. Sönmez (2005): "The Boston Public School Match," American Economic Review, Papers and Proceedings, 95, 368-371.

(2006): "Changing the Boston Public School Mechanism: Strategy-proofness as equal access," NBER Working paper, 11965.

AbdulkadiroĞlu, A., And T. Sönmez (2003): "School Choice: A Mechanism Design Approach," American Economic Review, 93, 729-747.

Balinski, M., And T. Sönmez (1999): “A Tale of Two Mechanisms: Student Placement," Journal of Economic Theory, 84, 73-94.

Baum-Snow, N., and B. Lutz (2011): "School Desegregation, School Choice and Changes in Residential Location Patterns by Race," American Economic Review, 101(7), 3019-3046.

Boustan, L. P. (2012): "School Desegregation and Urban Change: Evidence from City Boundaries," American Economic Journal: Applied Economics, 4(1), 85-108.

BPS (1999): "Modifications to Controlled Choice Student Assignment Plan, School Committee Order, November 10," Available from the archives of the Boston School Committee.

- (2012): "Introducing the Boston Public Schools 2012," Boston Public Schools, Available at: http://www.bostonpublicschools.org/register.

Budish, E., Y.-K. Che, F. Kojima, and P. Milgrom (2013): "Designing Random Allocation Mechanisms: Theory and Applications," American Economic Review, 103(2), 585-623.

Calsamiglia, C., G. Haeringer, and F. Kluin (2010): "Constrained School Choice: An Experimental Study," American Economic Review, 100(4), 1860-1874.

Connolly, J. (2013): "Testimony to School Committee, March 13," Available at: http://www.connollyforboston.com.

Crawford, V., And E. Knoer (1981): "Job Matching with Heterogeneous Workers and Firms," Econometrica, 49(2), 437-450.

DAley, B. (1999): "Plan Drops Race Role in Enrollment, Compromise Misses Point, Critics Say," Boston Globe, B1, October 20. 
EAC (2013): "Minutes from the Meeting of the Committee, January 14," External Advisory Committee, City of Boston, Available at: http://bostonschoolchoice.files.wordpress.com/2013/01/eac-minutes-1-14-2013.doc, last accessed: April 14, 2013.

Echenique, F. (2012): "Contracts vs. Salaries in Matching," American Economic Review, 102(1), 594-601.

Echenique, F., And B. Yenmez (2012): "How to Control Controlled School Choice," Unpublished working paper, Caltech.

Erdil, A., And H. Ergin (2008): "What's the Matter with Tie-Breaking? Improving Efficiency in School Choice," American Economic Review, 98, 669-689.

Erdil, A., And T. Kumano (2012): "Prioritizing Diversity in School Choice," Unpublished working paper, Washington University.

Ergin, H. (2002): "Efficient Resource Allocation on the Basis of Priorities," Econometrica, 70, $2489-2498$.

Gale, D., and L. S. Shapley (1962): "College Admissions and the Stability of Marriage," American Mathematical Monthly, 69, 9-15.

Goldstein, D. (2012): "Bostonians Committed to School Diversity Haven't Given Up on Busing," The Atlantic, October 10.

Haeringer, G., And F. KliJn (2009): "Constrained School Choice," Journal of Economic Theory, $144,1921-1947$.

Hafalir, I., B. Yenmez, and M. A. Yildirim (2012): "Effective Affirmative Action in School Choice," Theoretical Economics, forthcoming.

Handy, D. (2012): “Debate on Overhauling Boston Schools’ Assignment System Continues,” 90.9 WBUR, November 13.

Hatfield, J. W., And F. Kojima (2010): "Substitutes and Stability for Matching with Contracts," Journal of Economic Theory, 145, 1703-1723.

Hatfield, J. W., and P. Milgrom (2005): "Matching with Contracts," American Economic Review, 95, 913-935.

Johnson, C. R. (2013): "Speech to Boston School Committee, March 13," Available at: http://bostonschoolchoice.files.wordpress.com/2013/03/3-13-13-superintendent-sc-memoon-assignment.pdf, last accessed: April 14, 2013.

Kelso, A., and V. Crawford (1982): "Job Matching, Coalition Formation, and Gross Substitutes," Econometrica, 50, 1483-1504. 
Kesten, O. (2006): "On two competing mechanisms for priority-based allocation problems," Journal of Economic Theory, 127, 155-171.

(2010): "School Choice with Consent," Quarterly Journal of Economics, 125(3), 12971348.

KoJima, F. (2012): "School Choice: Impossibilities for Affirmative Action," Games and Economic Behavior, 75(2), 685-693.

Kominers, S. D., And T. Sönmez (2012): "Designing for Diversity in Matching," Boston College Working Paper.

MAPC (2013): "Recommendations to the External Advisory Committee on School Choice, February 25," Metropolitan Area Planning Council, Available at: http://www.mapc.org/BPSanalysis, last accessed: April 14, 2013.

Menino, T. (2012): "State of the City 2012," January 17, Available at http://www.cityofboston.gov/.

Ostrovsky, M. (2008): "Stability in supply chain networks," American Economic Review, 98(3), 897-923.

Pathak, P. A., and J. Sethuraman (2011): "Lotteries in Student Assignment: An Equivalence Result," Theoretical Economics, 6(1), 1-18.

Pathak, P. A., And T. Sönmez (2008): "Leveling the Playing Field: Sincere and Sophisticated Players in the Boston Mechanism," American Economic Review, 98(4), 1636-1652.

- (2013): "School Admissions Reform in Chicago and England: Comparing Mechanisms by their Vulnerability to Manipulation," American Economic Review, 103(1), 80-106.

Ravitch, D. (2011): The Death and Life of the Great American School System. Basic Books.

Roth, A. E. (1985): "The College Admission Problem is not Equivalent to the Marriage Problem," Journal of Economic Theory, 36, 277-288.

Seelye, K. Q. (2012): "4 Decades after Clashes, Boston Again Debates School Busing," October 4, New York Times.

Sönmez, T. (2013): "Bidding for Army Career Specialties: Improving the ROTC Branching Mechanism," Journal of Political Economy, 121(1), 186-219.

Sönmez, T., And T. Switzer (2013): "Matching with (Branch-of-Choice) Contracts at the United States Military Academy," Econometrica, 81(2), 451-488. 